2012-12-04

\title{
Effective Collaboration Through Multi User CAx by Implementing New Methods of Product Specification and Management
}

Vonn L. Holyoak

Brigham Young University - Provo

Follow this and additional works at: https://scholarsarchive.byu.edu/etd

Part of the Mechanical Engineering Commons

\section{BYU ScholarsArchive Citation}

Holyoak, Vonn L., "Effective Collaboration Through Multi User CAx by Implementing New Methods of Product Specification and Management" (2012). All Theses and Dissertations. 3489.

https://scholarsarchive.byu.edu/etd/3489

This Thesis is brought to you for free and open access by BYU ScholarsArchive. It has been accepted for inclusion in All Theses and Dissertations by an authorized administrator of BYU ScholarsArchive. For more information, please contact scholarsarchive@byu.edu, ellen_amatangelo@byu.edu. 
Effective Collaboration Through Multi User CAx by Implementing New

Methods of Product Specification and Management

Vonn L. Holyoak

A thesis submitted to the faculty of

Brigham Young University

in partial fulfillment of the requirements for the degree of

Master of Science

W. Edward Red, Chair

C. Greg Jensen

David T. Fullwood

Department of Mechanical Engineering

Brigham Young University

November 2012

Copyright (C) 2012 Vonn L. Holyoak

All Rights Reserved 


\title{
ABSTRACT \\ Effective Collaboration Through Multi User CAx by Implementing New \\ Methods of Product Specification and Management
}

\author{
Vonn Holyoak \\ Department of Mechanical Engineering, BYU \\ Master of Science
}

This thesis presents a new design process in which design specifications and task distribution are determined from a parallel multi user prospective. Using this method, projects are more easily decomposed into tasks that can be performed concurrently, thus decreasing the design time. Also, a framework is provided to determine the correct distribution of available talent and stakeholders that can be utilized on a given project. The research suggests that by involving the necessary stakeholders in a multi user setting, changes can be made quickly and without additional approval wait time. By including individuals from the various areas of required talent, persons of expertise will be able to work together in a mode of shared design rather than an iterative design process. Decreasing iterations as well as reducing wait time for approval will reduce the overall design time significantly. This method has been tested and validated utilizing controlled tests simulating real life situations of much larger scale. The validation results show that the new method does in fact improve design time and overall achievement of initial design goals

Keywords: multi user CAx, collaboration, design process, specification gathering, task decomposition, task distribution 


\section{ACKNOWLEDGMENTS}

Up to this point in my life, this document represents the culmination of many years seeking to better myself through education. As such it is only appropriate for me to acknowledge that I have not completed this journey alone, but have been supported and lifted up at every step. Throughout my life my parents, Vearl and Janie Holyoak have shown me by example that I can do difficult things. Amy, my beautiful wife is probably the one who offered the most support and inspiration to complete this work. She has ever been the patient, loving and gently prodding angel that I so desperately needed. My advisor Dr. Red, as well as Dr. Jensen and Dr. Fullwood have helped to guide my thoughts and give correction when needed. I have been greatly helped also by the financial support afforded by my work with the v-CAx Center. Above all, my Father in Heaven has been my support and led me through the trying times that all go through as we seek to improve. I thank you all, and shall endeavor always to be worthy of the great love and support you have shown me. 


\section{TABLE OF CONTENTS}

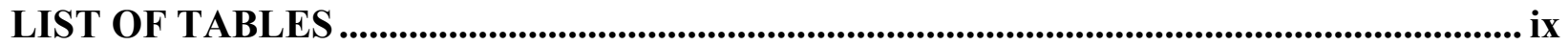

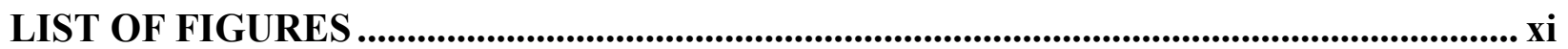

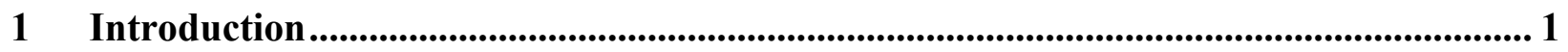

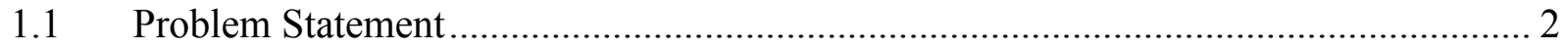

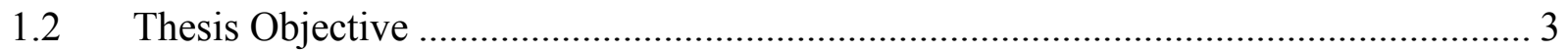

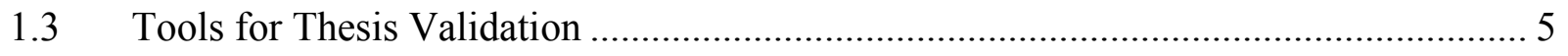

1.4 Document Organization ...................................................................................... 6

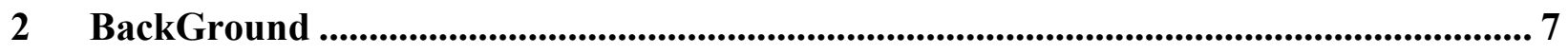

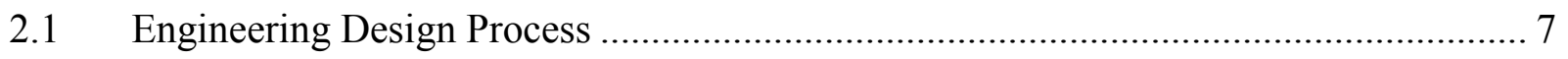

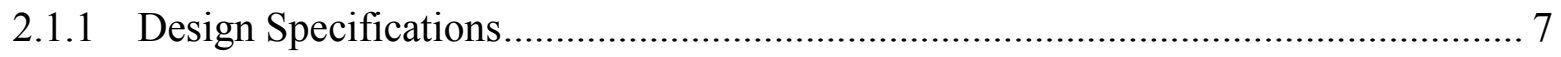

2.1.2 House of Quality ....................................................................................... 9

2.1.3 Design Tasks ........................................................................................ 10

2.2 Collaboration within Engineering Design ...................................................... 12

2.2.1 Decentralized Decision Making ................................................................... 13

2.2.2 Challenges and Solutions to the Decision Process .......................................... 15

2.3 Multi User Applications.............................................................................. 17

2.3.1 Independent Collaborative Applications ..................................................... 17

2.3.2 Transparent Adaptation for Converting Single User to Multi User Applications .... 18

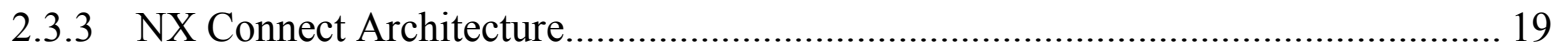

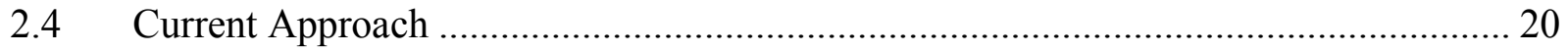

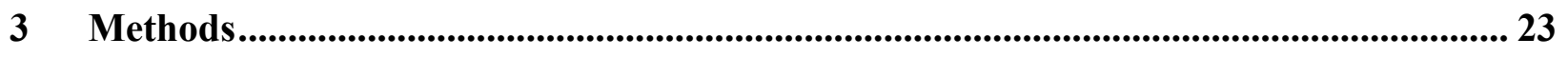

3.1 Overall Method for Collaborative Design ........................................................... 23 


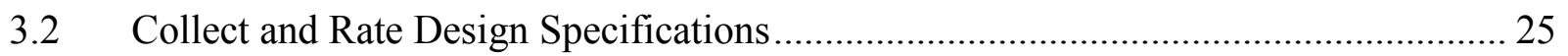

3.2.1 Determine Specifications to Fully Define the Project .............................................. 25

3.2.2 Required Talent, Importance, and Correlation Information Recorded ..................... 26

3.3 Decompose Project into Subtasks and Calculate Optimal Groups and Sequence........ 30

3.3.1 Specification based Project Decomposition............................................................ 30

3.3.2 Calculate the Task Relationships Matrix (TRM) ................................................... 31

3.3.3 Group Tasks based on Task Relationships and Calculate Optimal Sequence .......... 34

3.4 Compose Personnel Groups of Required Talent............................................................. 36

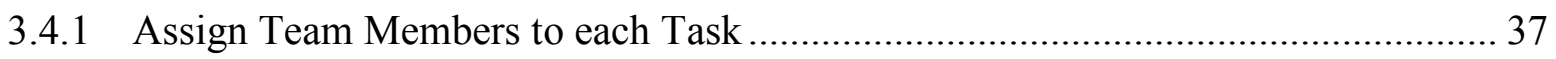

3.4.2 Group Talent Sets from Each Task into the Talent Group ...................................... 37

3.5 Talent Groups Perform Tasks Collaboratively within Multi user Environment .......... 37

3.5.1 Each User Participates Simultaneously On Each Task ............................................ 37

3.5.2 Communication of Design Rationale ..................................................................... 38

3.5.3 Each User Can Personally Make Changes to the Part .............................................. 39

$4 \quad$ Implementation ................................................................................................................................ 41

4.1 vManager - Product Development Process Software................................................. 41

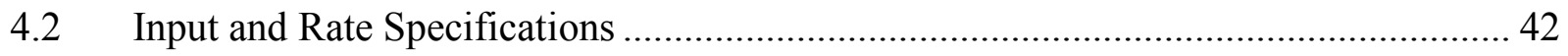

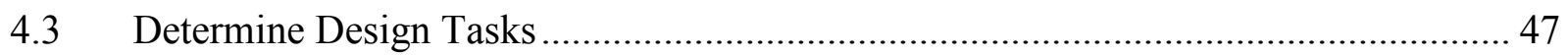

4.4 Implementation within NX Connect and Siemens NX 6.0 ...................................... 52

4.5 Increased NX Connect Functionality ....................................................................... 54

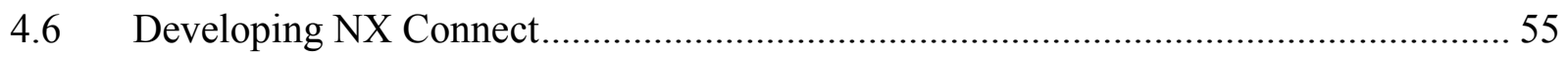

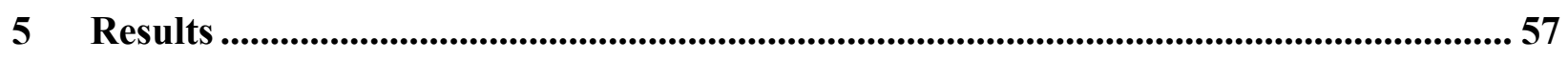

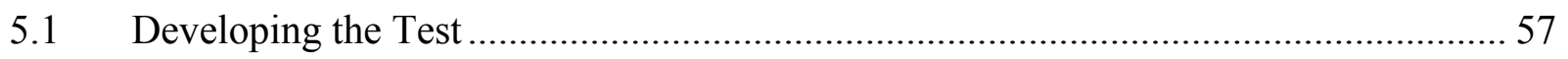

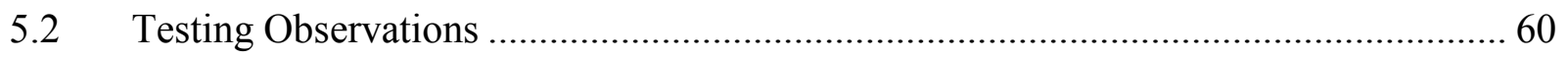

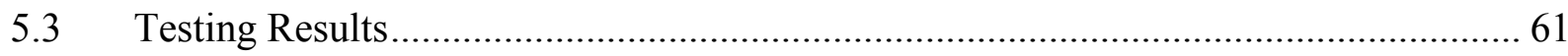


6 Conclusions \& Suggestions For Future Work....................................................................... 65

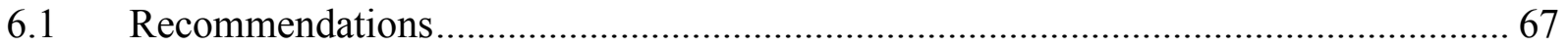

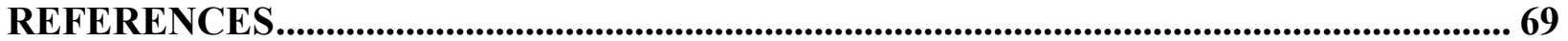




\section{LIST OF TABLES}

Table 5-1: Single User - Iterative Design Test Results..............................................61

Table 5-2: Multi User Collaborative Design Test Results..............................................62 


\section{LIST OF FIGURES}

Figure 2-1 Sample House of Quality (Lowe \& Lowe, 2011) .........................................10

Figure 2-2 Simple Design Structure Matrix................................................................ 11

Figure 2-3: Simple interdependent design problem...................................................... 14

Figure 3-1: Example Specification Rating Matrix ..................................................28

Figure 3-2: Sample Specification Importance Ratings ...............................................29

Figure 4-1: vManager Input Specifications Tab .....................................................43

Figure 4-2: vManager Assign Talent Tab ..............................................................44

Figure 4-3: vManager Rate Importance Tab..........................................................45

Figure 4-4: vManager Specification Correlations Tab ...................................................46

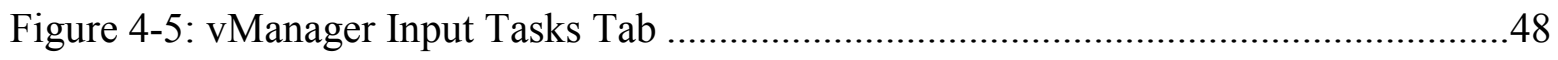

Figure 4-6: vManager Task Relationships Tab.......................................................49

Figure 4-7: vManager Design Groups Tab ...........................................................50

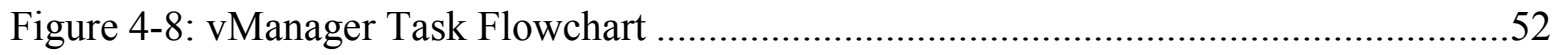

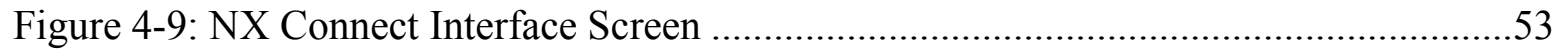

Figure 4-10: NX 6.0 Running NX Connect ..........................................................54

Figure 5-1: Single User Design Test Sample Images ................................................63

Figure 5-2: Multi User Collaborative Design Test Sample Images...................................64 


\section{INTRODUCTION}

The goal of any engineering design company is simple; to achieve a successful product. The level of success of a completed product can be measured in a variety of ways, but the most common is by analyzing three main factors: cost, performance, and completion time (Lock, 2007). The difficulty arises in the fact that these factors are not independent of each other. Tradeoffs must often be made. For example, often as a result of making improvements to a products performance, costs will increase due to additional manufacturing costs or a more rigorous design process. Also, completion time and cost are usually directly related as the commonly used phrase "time is money" states. Designer wages, facility rental or upkeep, and design tool expenses all contribute to rising costs as the design process lengthens. In addition, “developing products faster than competitors can increase market share, profit, and long-term competitive advantage (Ford \& Sterman, 2003)." Designers must decide which of the factors are the most important to each product and move forward with the design.

Recent research into multi user computer aided engineering (CAx) tools has provided preliminary prototypes allowing multiple designers to enter and work in the same design space simultaneously (Mishra, Varshney, \& Kaufman, 1997) (Red, Holyoak, Jensen, Marshall, Ryskamp, \& Xu, 2009). It is the hope of these researchers, that by allowing multiple users to work in parallel on the same model, that design completion time will improve. However, "technology alone does not make for a successful collaboration effort; it really requires a 
combination of process, culture, and technology to be able to gain the greatest amount of business value from your collaboration efforts (Byrch, 2010).” By also implementing project management techniques designed specifically for use with multiple CAx users, improvements are made in cutting completion time, improving product costs, as well as increasing product performance through increased collaboration and knowledge sharing.

\subsection{Problem Statement}

Design activity is a creative process "in which there exists an element of the unknown, such as lack of information or incomplete knowledge of the relationships among issues". Design cannot be reduced to simply following a prescribed algorithm or calculation to arrive at the end goal, but is a human activity where actual, perceived, and potential conflicts are identified and resolved. (POHL, CHAPMAN, \& POHL, 2000) While much effort has been made to provide intelligent and easy to use design tools, they remain just tools, leaving the burden on the designer to create viable solutions.

It is common to decompose design activities into smaller design spaces to be worked separately by individual designers. These individual designs are then merged as a cohesive solution to the original problem. However, unlike algorithms that can be decomposed and solved piecewise, conflicting requirements and boundary conditions make design space decomposition difficult without the tools to communicate information and collaborate intent. Conflicts arise that add significant complexity to each design space.

Prevailing multi user optimism holds the belief that including more designers in the same environment will decrease the amount of design time. Wooley, et all, provide statistical evidence suggesting that groups of individuals tasked with solving problems are more effective than any one of the individuals within the group. (Woolley, Chabris, Pentland, Hashmi, \& 
Malone, 2010). However, current CAx tools are usually very complex in nature and are built upon a single user, serial process type architecture. Even in a single user environment, design conflicts are not uncommon and increase significantly when designs are not carefully planned out. Applying multi user collaboration within the current CAx tool architecture magnifies the potential for design conflicts, possibly increasing dramatically with each distinct user in the design session (Feng \& Chen, 2006). These conflicts will necessitate an enormous amount of collaboration in the form of communication, data sharing, and compromise between designers. Without an organized team of designers with exceptional proficiency in collaboration, a reduction in design effectiveness and/or increase in the duration of design will occur. Without additional controls or management structure, allowing multiple designers to work simultaneously would likely be counterproductive.

\subsection{Thesis Objective}

As the objective of my research, I have developed a method to manage multiple collaborators within the same design space with the goal of improving design in each of the three main factors for project success: cost, performance, and completion time.

To accomplish this task a study was performed to examine current project development processes and to identify possible areas where improvements can be made by implementing multiple user technology.

In the initial design stages of a complex part, design specifications are based on customer requirements and existing technology. Between these specifications varying levels of dependency and tradeoffs are often inherent when setting the target values for each specification. These specifications are revisited various times throughout the initial product design phases and 
due to the tradeoffs between the specifications, often undergo various iterations to arrive at a final set of target specifications.

Also, a critical point in the early design process is that of task decomposition. "Many design challenges are too complex to solve as a single problem and can be usefully divided into several simpler sub problems (Ulrich \& Eppinger, 2004, p. 101)." Among the various forms of decomposition that exist, the methods of functional decomposition or decomposition by key customer needs (specifications) were the most relevant to this research.

Particularly, in large complicated products the decoupled tasks have complex interdependent relationships which add difficulty into the design management. As with developing target specifications, the process to complete tasks and resolve challenges due to these interdependent relationships generally requires an iterative process. Iterative processes can be very time consuming and often unpredictable, causing many design tasks to overshoot target completion dates. "Iteration cycles can delay projects by being more in number, longer in distance which information must travel, slower in traversing that distance, and occurring later than possible (Zhang, Qiu, \& Zhang, 2006).”

Much of the problem with the iterative process can be traced to the various stakeholders and design personnel who must review the information, and after modifying and providing input pass it on to the next designer in the cycle. This transfer of information can be inefficient if each reviewer has other schedule commitments. The resulting communication delays between reviewers make it difficult to resolve conflicting requirements.

As a result of studying the engineering design process, it was determined that implementation of multi user tools can have the most beneficial results when focused on reducing iteration cycles and cycle time. This can be achieved by incorporating designers with 
the specific skill set most suited to perform each design task as well as all personnel required to make decisions or give approval. It is also proposed that each designer simultaneously participates fully in all design decisions. By implementing multiple users in this way, the distance information must travel will be virtually eliminated, and iteration cycles able to be completed instantaneously, as each member of the iteration process is present in the design session.

To accomplish this objective, this research will present a method to determine a set of task groups derived from the project tasks and organized using the target specifications as criteria. These task groups will be linked to a list of the required areas of expertise, or talent, the designers will need to fully complete each task in the respective task group. Within the task groups each task is sequentially optimized to allow for the multi user design group to collaborate together in a fast, efficient, more productive manner.

\subsection{Tools for Thesis Validation}

To validate the method created through this research various engineering tools were used. The CAx application used the Siemens NX 6.0 CAD system. A prototype multi user add-on NX Connect was utilized and modified to perform necessary testing. Initially NX Connect was a proof-of-concept showing the feasibility of providing multiple users in the same commercial CAD software environment. Modifications for the purpose of this research have developed this add-on into a much more robust and useable application to perform multi user design.

A management tool named vManager has been created to allow the project manager to go through a step-by-step graphical interface to input design specifications, determine design tasks, and rate each of these based on interdependencies. The vManager software in turn will provide 
the project manager with a list of task groups, sequence of tasks within those groups, and the required talent (designers of specific fields or functional department) for each of the task groups.

\subsection{Document Organization}

This research builds off the efforts completed by others in the fields of project management, task decomposition, and multi user applications. This prior research is presented in chapter two along with an explanation of how the current research builds on these efforts. Chapter three presents the generalized method to improve collaboration through specialized multi user design groups. Chapter four describes the tools created to implement and test the research method. The results of a group of tests are presented in chapter five that provide evidence of improvement to the design process when following this method. Finally, chapter six

presents the conclusions drawn from the research and subsequent tests. Also, possible areas for future work are suggested in this final chapter. 


\section{BACKGROUND}

This research explores approaches to collaborative decision making and the application to product design and evolution, while also considering collaborative tools that will aid in the design process. The following is a review of current literature dedicated to the engineering design process, collaboration and decision making, and the evolution of multi user software applications.

\subsection{Engineering Design Process}

The engineering design process has been around for a long time, and while virtually every organization employs slight variations to this process, the core pieces of the process have remained fairly consistent. The design process exists to help companies conceive, design, and commercialize a product in the most efficient way possible. Having a well-defined development process helps to boost overall quality, define the roles of the development team, and provides opportunities to improve the process through observation of potential problem areas (Ulrich \& Eppinger, 2004, p. 13).

\subsubsection{Design Specifications}

Key to the early stages of the design process is the gathering of customer requirements and translating those requirements into specifications. Customer requirements are often broad or 
subjective statements that can be interpreted a variety of different ways. In contrast, a set of product specifications is intended to completely define the project in quantifiable, measurable values. Even with the advances in engineering tools to help with this phase, the manner in which target specifications are determined is largely subjective. The design team owns the responsibility to set target specification values based on the statements of a customer, financial considerations, and technological limitations (Ulrich \& Eppinger, 2004, p. 72).

As the design team attempts to set target specifications tradeoffs become apparent. One example is set of tradeoffs that exist between cost, strength, and weight of a new bicycle design. To improve the strength of the bicycle frame you can either make the material walls thicker, thus adding weight to the bike, or you can use materials with a greater strength to weight ratio, which will drive the cost to produce the bicycle higher.

Because of the complexities of balancing the many tradeoffs that are common in an engineering product it becomes necessary to review and update the target specifications multiple times in the early design process before a final set of specifications is achieved. The initial specification for the bicycle may not take into account the cost, strength, and weight tradeoffs, but list an ideal value for each of these figures. As the design team researches the concepts and technologies available it must return to the specifications and adjust the specifications to more realistic values. This is accomplished by reviewing the customer needs the specifications were derived from, and weighing the relative importance to the customer of each respective need as well as the relative cost of improvement each specification will have based on the other conflicting specs. This is often an iterative process as the design team searches for the optimal set of target specifications. 


\subsubsection{House of Quality}

One common tool to aide in the development of design specifications is known as the house of quality (HOQ). The HOQ is a way of visualizing the relationships between customer requirements and product specifications as well as the design priorities and specification interdependencies (Hauser \& Clausing, 1988). It integrates various product stakeholders such as marketing, design engineering, manufacturing and others into the process of translating design requirements into specifications. A sample house of quality is shown on the following page.

In this example the specifications are being developed for a climbing harness. Each customer requirement is listed down the left side of the figure and grouped into similar categories. Just to the right of the customer requirements is a customer importance rating that determines the relative value of each customer requirement as opposed to the others. Across the top written vertically are the chosen design specifications in this case they are labeled technical requirements. The purpose of the body or mid-section of HOQ is to rate the relationship of each customer requirement to each specification. These relationships together with the customer importance will determine the relative importance of each specification. Finally, the roof of the HOQ is used to identify how the specifications correspond to each other whether in a positive or negative manner (Lowe \& Lowe, 2011).

Utilizing a modified form of the HOQ, Park and Kim developed a method to determine an optimal set of design requirements. Unlike in conventional HOQ matrices that do not incorporate the specification correlations as found in the roof when calculating specification values, they believe that due to the often closely linked nature of the specifications it is very important to take the correlations into account (Park \& Kim, 1998). These correlations take on even further meaning when planning for multi user design. 


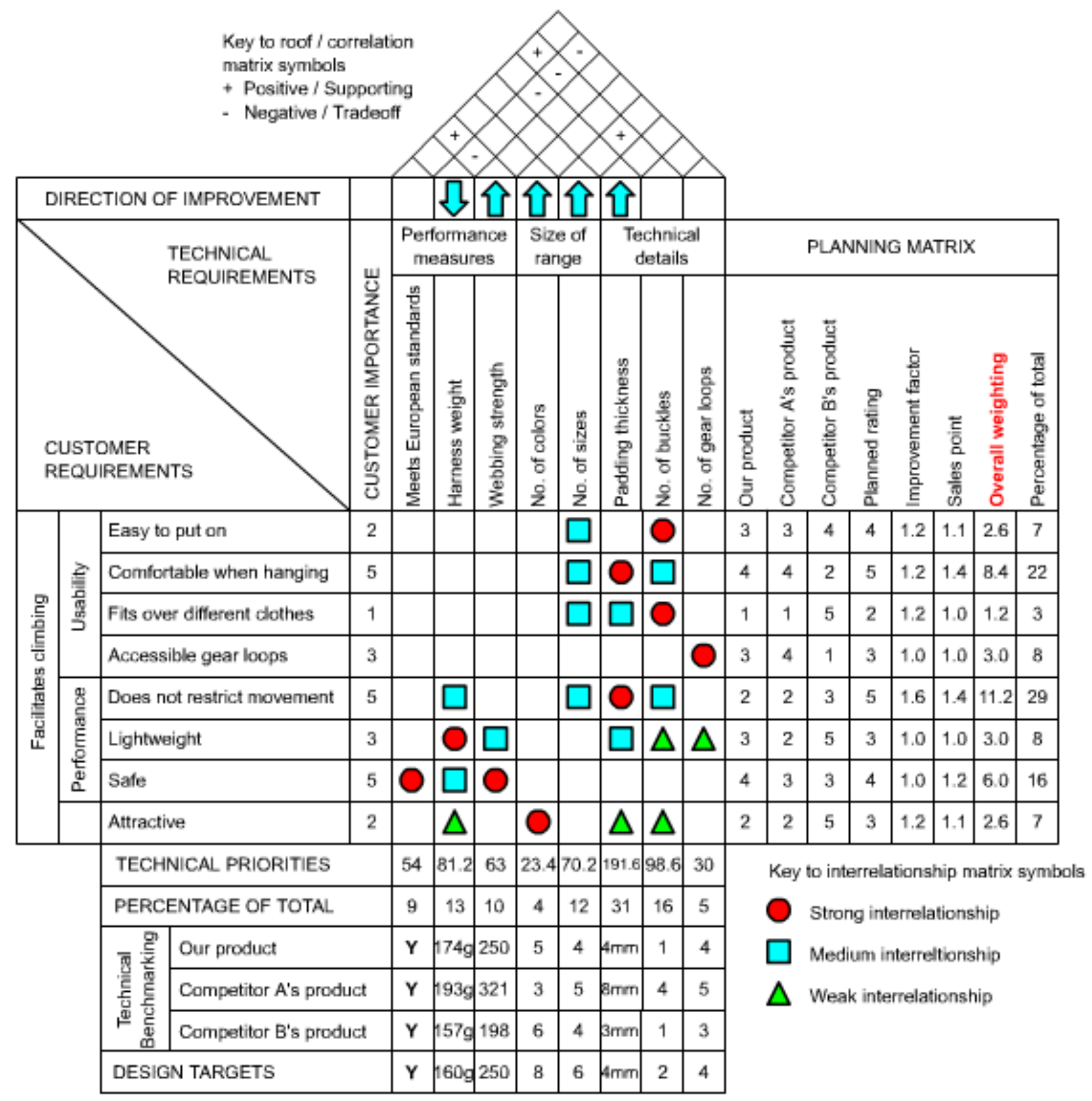

Figure 2-1 Sample House of Quality (Lowe \& Lowe, 2011)

\subsubsection{Design Tasks}

Once the design specifications have been developed, they can now be utilized in problem decomposition and the determination of project tasks. At the design task level there exists yet another layer of interdependencies. Each defined task will have corresponding coupled task 
strength to the other defined tasks. According to Tang and coauthors, there exist three types of relationships between design tasks: uncoupled, coupled, and decoupled (Tang, Zheng, Li, Li, \& Zhang, 2000).

Uncoupled relationships signify that the interdependency between two tasks is extremely low allowing those tasks to be performed in parallel. Coupled relationships denote very high interdependency where design decisions affect each task simultaneously. These tasks generally require iterations to resolve and must be executed concurrently. Decoupled relationships mean that the decisions made for early tasks will propagate down to later tasks; however there is little or no correlation between decisions made on later tasks that reflect back to the tasks previously completed. This indicates that the tasks should be performed in sequence (Tang, Zheng, Li, Li, \& Zhang, 2000).

The Design Structure Matrix (DSM) is an engineering tool to help to identify the differing types of relationships that exist within each task. A sample of the DSM is below.

\begin{tabular}{|c|c|c|c|c|c|c|c|}
\hline Task & A & B & C & D & $E$ & $F$ & G \\
\hline A & 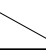 & & & & & & \\
\hline$B$ & $X$ & $\gamma$ & & & & & \\
\hline$C$ & $X$ & $x$ & 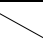 & & & & \\
\hline$D$ & & & $X$ & 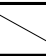 & & & \\
\hline$E$ & & $X$ & & $X$ & & $X$ & \\
\hline$F$ & & & $X$ & & & 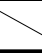 & $X$ \\
\hline G & & & & & & $x$ & $\gamma$ \\
\hline
\end{tabular}

Figure 2-2 Simple Design Structure Matrix

Each Task is represented both on the left hand side of the table as well as across the top. For a simple matrix like the one shown, an $\mathrm{X}$ is placed on the square when the task located on the corresponding row is dependent on the task in the column. From the above figure we can see 
that task A through D must be done sequentially, as each task is dependent on the completion of the previous task. However, task F can be performed in parallel with task D. Also we note that task $\mathrm{E}$ and task $\mathrm{F}$ have a dependency above the diagonal. In the case of task $\mathrm{E}$ we can easily rearrange the task order and place it after task F; however we see that F and $\mathrm{G}$ are interrelated and would ideally be performed concurrently.

Tang and coauthors develop an algorithm to recognize the coupled activities and then to re-engineer the design tasks to provide for a more concurrent approach (Tang, Zheng, Li, Li, \& Zhang, 2000). Zhang and coauthors build upon this approach and develop an optimal sequence of tasks. They provide a more objective approach to determining task dependencies by basing the relationships of the related design parameters (Zhang, Qiu, \& Zhang, 2006). Zhang and coauthors assume iterations must be performed and base their optimized task sequence on the best path through required iterations. This research attempts to minimize iterations by providing all the needed talent in the design session therefore requiring a different approach than that used in previous papers.

\subsection{Collaboration within Engineering Design}

Beginning in the mid to late 80 's concurrent engineering has become increasingly more important to the design process. (Imai, Nonaka, \& Takeuchi, 1986) This push into concurrent engineering has facilitated an increase in globalization of today's design companies as well as escalating levels of complexity of engineering products. Concurrent engineering has become more essential than ever in the engineering design process. "(Concurrent Engineering) is a philosophy that suggests the need to consider design issues simultaneously where they were

considered sequentially in the past (Tang, Zheng, Li, Li, \& Zhang, 2000).” Communication and collaboration become key contributors to the success of concurrent engineering processes. 
Despite much research in the area of engineering collaboration, many shortcomings have yet to be overcome in the collaboration methods and tools available (Ryskamp J. D., 2010, p. 5). Red surveys much of the available literature regarding the use of engineering tools and provides key insight into these existing shortcomings (Red, Holyoak, Jensen, Marshall, Ryskamp, \& Xu, 2009). Key tools used by engineers in the design process exhibit large weaknesses in collaborative design. CAx tools for example have been developed almost exclusively for single users with a largely serial process in mind. This is evidenced by file management software that requires a user to check out a given file to ensure that user is the only one performing modifications.

Other shortcomings include an increased security risk as intellectual property is shared widely through collaboration as well as new challenges in the area of conflict management and decision making.

\subsubsection{Decentralized Decision Making}

Among the previous shortcomings, the process by which design decisions are made is of particular importance. "Collaborative design involves various stakeholders with different intentions, backgrounds, and circumstances. The group design activities are influenced not only by technical decisions, but also by social interactions. Due to the various dependencies that exist within any design, conflicts are a certainty. (Lu, Cai, Burkett, \& Udwadia, 2000).” With such a diverse and often conflicting group of decision makers, arriving at a final design can often be difficult and extremely time consuming.

Panchal notes two types of decision making strategies; centralized and decentralized decision making (Panchal, Fernandez, Paredis, Allen, \& Mistree, 2007). Centralized decision making consists of a single decision maker who receives knowledge and direction from a number 
of different experts in their respective areas. This solution generally allows for a quicker resolution to the challenges, but at the expense of requiring one who is not an expert in the various necessary areas to make the final decision possibly resulting in an inferior final product.

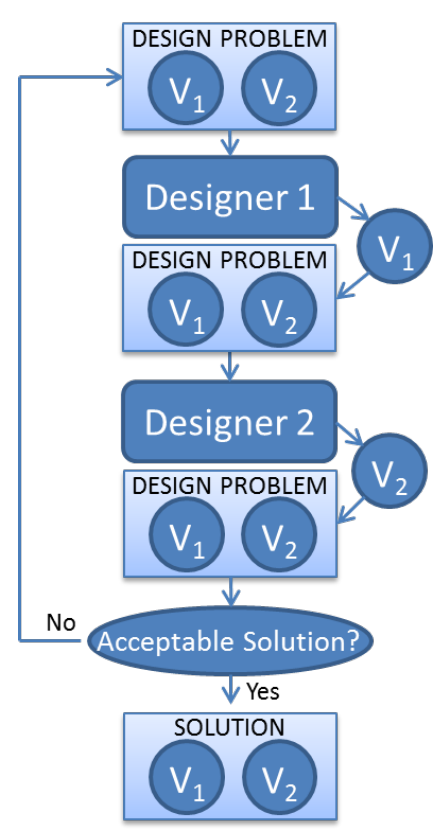

Figure 2-3: Simple interdependent design problem

Decentralized decision making distributes the decision responsibility to persons who specialize in their respective areas. "Decentralized decisions are generally coupled by nature and require a two-way flow of information between decision-makers as well as active involvement of domain experts throughout the decision-making process (Panchal, Fernandez, Paredis, Allen, \& Mistree, 2007)." Chanron and Lewis state "The decentralization of decisions is unavoidable in a large organization where having only one centralized decision maker is usually not applicable. A more effective way is to delegate decision responsibilities to the appropriate person, team, or supplier (Chanron \& Lewis, 2003). 
Within a decentralized decision making process, complex problems are broken down into multiple sub problems that are smaller and less complicated. Ideally, these problems could be solved on an individual basis, but the majority of the cases involve design variables and parameters that influence the design of more than one subsystem. A common example of this process using a simplified model of two designers each with responsibility for a single design variable is as follows. Designer 1 will determine a solution that corresponds to his/her design requirements and then will pass the solution on to designer 2. Designer 2 will then make changes to solve his/her corresponding problem at which time the design will be passed back to designer 1 and the process restarts until a solution is found that is satisfactory to each decision maker (Chanron \& Lewis, 2003). This method is dependent upon design iterations to arrive at an optimal solution which is often inefficient and time consuming.

\subsubsection{Challenges and Solutions to the Decision Process}

One of the main problems associated with design iterations is the amount of time it takes to transfer information from one designer to another. Chanron and Lewis explain that "even within the same corporation, perfect information and cooperation is difficult to achieve due to several factors, including the complexity of the design, geographical separation or information privacy (Chanron \& Lewis, 2003)."

Research to improve the decentralized decision making process has focused on how to speed up the design iteration process by improving information transfer, and by reducing the number of iterations to arrive at the optimal solution. Panchal and co-authors support an interval based constraint satisfaction method in which designers pass an interval of acceptable values as opposed to single point approach (Panchal, Fernandez, Paredis, Allen, \& Mistree, 2007). Liker and co-authors address the value by using this method to improved communication. "A set- 
based approach allows team members to communicate information they actually possess in the early stages of the design process, rather than sharply defined decisions that they cannot be confident will survive (Liker, Sobek II, Ward, \& Cristiano, 1996).” Their research also strongly suggests that using this method improves overall completion time through this improved communication as well as reduced rework.

To arrive at a solution faster, researchers have applied techniques such as game theory (Marston \& Mistree, 2000). Game theory is valuable within decentralized decision making as each designers decisions has a varying amount of impact on each of the other decision makers. This takes place in an environment where communication is usually not optimal. Marsto and Mistree showed that by using game theory in the decision making process, a set of feasible solutions are produced which allows the designers to quickly narrow their efforts to a smaller set of solutions. However, various limitations still exist. It is difficult to deal with both continuous and discrete design variables; there is uncertainty in the solution outcomes, and it is difficult to represent more than two players. Also levels of cooperation between the designers vary within the design process and calculating the potential outcome is difficult (Marston \& Mistree, 2000).

Despite the efforts made to improve the decision making process, it is still very time consuming due to iterative cycles. This research attempts to directly address the problem of wasted time and energy on lengthy iterations by bringing all necessary stakeholders together in the same design space to simultaneously work and oversee the work of others. While not eliminating the iteration process it will close the communication gap and often allow for immediate conflict resolution. 


\subsection{Multi User Applications}

Over the past few decades Computer Aided Design (CAD) tools have become the primary tool for engineering design. Advances in these tools have improved design processes effectively with more accurate and reproducible documentation. However, with the rise of the CAD systems, which are almost exclusively single user serial design tools, design collaboration has been limited (Red, Holyoak, Jensen, Marshall, Ryskamp, \& Xu, 2009).

To address the problems of organizing the immense amount of information generated by CAD and other engineering tools, product lifecycle management (PLM) tools began to emerge in the late 1980’s (Sääksvuori \& Immonen, 2008). PLM helps address problems associated with multiple designers working on a single design by providing a framework to manage ownership and changes to design files through a means of a checkout/check-in procedure and revision control. These tools are valuable to help collaborators avoid serious design conflicts, however, they do little to bring designers together and foster better communication and concurrent design.

\subsubsection{Independent Collaborative Applications}

As technology has improved a strong push is being made toward true multi user applications. Well-known examples of these multi user applications are the spreadsheet, word processor and presentation applications within Google Docs (Google Docs, 2011). These applications allow for multiple editors to freely make changes within a single document simultaneously without any additional constraints. Collaborative applications are proving to be both popular and useful as Google Docs applications have been gaining ground in the commercial world as more and more companies signing up (Lohr \& Helft, 2007).

This collaborative technology has been extended into the CAx tools for engineering where designers can work simultaneously in the same file (Fuh \& Li, 2005). However, CAD 
systems are complex with a wide variety of functions each user can utilize making the prospect of extending it to multiple users very difficult. $\mathrm{Li}$ and coauthors believe that a fundamental change in many aspects of CAD systems including infrastructure design, communication algorithms, and geometric computing algorithms are necessary (Li, Lu, Fuh, \& Wong, 2005). Perhaps because of this complexity, the majority of multi user CAD prototypes are entirely new tools built from the ground up (Ryskamp, Jenson, Mix, \& Red, 2010). Examples of these prototypes include COCADCAM (Kao \& Lin, 1998) CollabCAD (Mishra, Varshney, \& Kaufman, 1997) and CyberCAD (Tay \& Roy, 2003).

\subsubsection{Transparent Adaptation for Converting Single User to Multi User Applications}

In contrast to entirely new multi user applications; Sun and coauthors presented the method of transparent adaptation (TA) that sets forth techniques to convert single user tools into collaborative applications. "The TA approach is based on use of the single-user application's API (application programming interface) to intercept and replay the user's operations, so it requires no access or change to the application's source code (thus being transparent) (Sun, Xia, Sun, Chen, Shen, \& Cai, 2006)." Through this method a collaborative version of Microsoft Word and Microsoft PowerPoint were developed.

Zheng and coauthors extended the transparent application method to engineering tools by adapting AutoCAD a single user CAD system to a collaborative design tool (Zheng, Shen, \& Sun, 2008). The software tool utilized for this research, NX Connect, has also utilized this method. 


\subsubsection{NX Connect Architecture}

In order to perform this and other related collaborative research, a prototype collaborative CAD system was developed as an add-on to the commercially available CAD software package Siemens NX. NX was chosen because of the widespread use within industry, availability to the author and fellow researchers, as well as access to an extensive API.

NX Connect allows multiple users to open and actively make changes to a part file simultaneously. Each user is able to view the part independently and utilize the zoom and rotational functions without affecting the view of the remaining users. This allows each user to focus on the intended task while viewing updates made to the part file by other users.

NX Connect is made up of four separate modules: information storage module, data capture module, data sync module, and the NX controller. The information storage module alone resides on a server with the remaining three on each client or individual user's computer. The information storage module is database that holds all information about each CAD part.

One of the main concerns of a collaborative environment is the ability to quickly send and receive data without a significant lag between users. This was achieved by breaking each part into basic parameters such as floats, integers, or strings. These parameters are referred to as primitives and are the only form of data stored in the database.

The NX controller is responsible for both sending and receiving data from the database. The controller will push the primitives of each recorded action done within NX to the database in an organized manner to be able to retrieve that data on other user's computers and reconstruct the

performed action. Each client utilizes a controller to gather the database information and performs each action locally. 
The data capture module is responsible for watching the local NX session to determine if an action was performed. It then filters the performed actions to determine if they are to be recorded and sent to the database. Then controller is then notified if the action is to be recorded and the data is sent.

In contrast, the data sync module watches the database to determine if changes have been made. It will then notify the controller to pull the data from the database and recreate the action on for each user.

This architecture has proven to work well but with some limitations. Due to the storage method chosen, each feature available within the CAD software tool must be individually implemented. This becomes an extremely long process to include a large subset of the Cad system's functionality. Also, some actions available within the CAD system as a single user application prove to be especially difficult to implement in a multi user environment such as an undo function.

NX Connect does not in any way interfere with the user interface of Siemens NX. A separate window is utilized to load parts from the data base or to save the current part to the database, but all modeling tools are selected from within the native graphical interface (Red, Holyoak, Jensen, Marshall, Ryskamp, \& Xu, 2009).

\subsection{Current Approach}

This research looks to build on all the previous research by utilizing and modifying many of the above mentioned methods. Initially, a modified form of the HOQ is employed to determine parameter importance and correlation. A modified form of a DSM will then be utilized, generated with the specification relationship data, providing a more advanced tool basing the task relationships on the driving specifications for each task to develop a series of task 
groups to be performed in parallel. Within each group tasks will be completed in a serial or concurrent fashion. Also, based on input from the project manager at the task level, a required talent list will be generated for each group indicating what designers and other stakeholders need to participate in the design sessions. Each member of the design groups will work together in the same CAx design environment to perform the task list outlined. 


\section{METHODS}

This chapter describes the methods used to improve the design process by providing a framework to guide the collaboration of multiple users within a design session. The proposed method is intended for design of complex parts where the associated specifications intersect a wide variety of backgrounds. Such parts are overly complicated for a single designer based on the many product requirements. The method will be presented verbally, graphically, and mathematically to improve understanding. Figures used for demonstration will include screen images of the CAD software NX 6.0 as well as the multi user management software vManager developed for this research. Further details regarding the method implementation are addressed in Chapter 4.

\subsection{Overall Method for Collaborative Design}

At the onset of any design task, a project manager or project management team is selected to organize the users who will be collaborating, and fully define the goals and intent of the design. Specific responsibilities of the management team are outlined as part of the overall method.

The proposed method for improving collaborative design is organized into the following steps, each of which will receive further discussion later in the chapter.

\section{Collect and rate design specifications}


i. The management team will determine a set of design specifications or requirements to fully define a project.

ii. Each specification is analyzed by determining correlation factors between specifications, relative importance to the design as a whole and required talent to fully address the specification.

iii. Absolute importance will be calculated based on relative importance and correlation factors.

\section{Decompose project into discrete subtasks and calculate optimal completion} groups and sequence.

i. The project is decomposed into discrete design tasks utilizing the information gathered for the design specifications.

ii. Based on correlations between Specifications task relationship values are calculated.

iii. Based on relationships between tasks, tasks are grouped and organized into an optimal sequence.

\section{Compose personnel groups of required talent.}

i. Each task will be given a required team that is necessary to determine all decisions associated with the task.

ii. For each task group, a corresponding personnel group will be assigned consisting of required talent to perform all tasks within the group in the optimal sequence.

iii. Output design sequence flow chart to demonstrate the design group work order and dependencies. 
4. Each talent group will perform corresponding design tasks within a multi user CAx environment.

i. Tasks are to be performed in the prescribed manner with each required team members participating simultaneously to complete each task.

ii. Team members will communicate important design decisions and rationale and all will have an understanding of the purpose behind each decision made.

iii. All users have equal ability to edit the design part to meet their respective design requirements.

\subsection{Collect and Rate Design Specifications}

For any design task it is critical to gather all the required specifications to fully define the end goal of the project. To best organize a specific project to take advantage of the benefits of multi user concurrent design it is necessary to begin in the early stages of product development. Each specification is considered individually and assigned the necessary talent required to understand and achieve the required target values, or to make compromises when design conflicts make this a necessity. Determining the personnel involved in decision making with respect to each specification will ensure that for the tasks that will later be constructed from these specifications, all necessary decision makers will be involved.

\subsubsection{Determine Specifications to Fully Define the Project}

This important step in the design process is often done by gathering customer requirements as well as performing market research. This information, often vague and open to various interpretations must then be translated into specifications that will completely define the 
scope of the project. Tools such as the House of Quality help ensure an acceptable set of specifications. This step must be performed by the project manager or a team that the manager assembles for the task.

\subsubsection{Required Talent, Importance, and Correlation Information Recorded}

For each specification generated to define the project it is now important to supply additional information to be used later in the organization of multi user groups and tasks. This additional information consists of the expertise or talent necessary to successfully design for each specification, the relative importance of each specification compared to all others, and the correlation between each of the specifications.

Talent refers to the area of expertise or responsibility within the company that a product designer possesses. For example, a company may operate with three separate departments: Mechanical Engineering, Manufacturing, and Marketing and Sales. When a new product is to be designed it is likely that each of the departments will contain the necessary expertise to make decisions on one or more specifications that define the product. In this simplified example let us suppose the company is designing a boat. The designer representing the mechanical engineering department would be responsible to choose a material best suited for the design. The marketing group would make decisions on specifications that relate to aesthetics. And some specifications such as the overall size and shape might require input from each of the groups. The mechanical group to ensure the necessary strength and buoyancy, the manufacturing group to determine ease and cost of manufacturing, and the marketing group to ensure the size and shape would appeal to potential customers. It can be seen that the required talent for each specification can consist of one or multiple different areas within the design company. 
The project management team is tasked with the determination of the required talent for each specification. The management team must take into account all the necessary information and expertise a designer or group of designers must have to be fully qualified to take ownership of the corresponding specification.

Next, the manager will determine correlations between each of the individual design tasks. This determination of correlations usually corresponds to the roof portion of the House of quality (Hauser \& Clausing, 1988). Each specification is to be evaluated individually against all other design specifications to determine its set of correlation values. As a specification is evaluated, the manager is to determine how much the current specification is affected by a change in each of the other specifications. This implies that a set of two specifications will have two separate values defining the relationship between the two. One value will correlate how the first is affected by the second, and the next value will correlate how the second is affected by a change in the first. This research builds on the concept of a DSM matrix utilizing a scored version called a specification rating matrix (SRM) and is shown on the following page.

The rows represent a specification that is in a state of modification. Each cell in that row corresponds to different specification shown along the top. The value is determined by defining how the modifications made to the specification on the left would impact the specification listed above. As shown in the figure, Specification A and B are closely related to each other while C and D have little or no correlation. Note that Specifications C and D are moderately affected by $\mathrm{B}$; however B is affected little by a change in either C or D. 


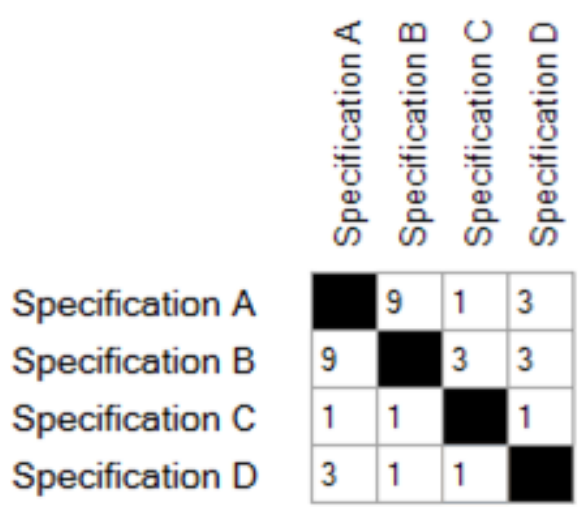

Figure 3-1: Example Specification Rating Matrix

The rows represent a specification that is in a state of modification. Each cell in that row corresponds to different specification shown along the top. The value is determined by defining how the modifications made to the specification on the left would impact the specification listed above. As shown in the figure, Specification A and B are closely related to each other while C and D have little or no correlation. Note that Specifications C and D are moderately affected by $\mathrm{B}$; however B is affected little by a change in either C or D.

The chosen rating scale is a 1-3-9 scale. The greatest correlation between two specifications corresponds to a nine, moderate correlation corresponds to a three, with one denoting little or no correlation. This scheme was chosen after a preliminary survey was performed to determine the value of a continual rating scheme as opposed to a discrete value scheme. The continual value rating system proved to be more difficult and significantly more time consuming for the manager to complete than the discrete value method. Also, when reviewed again, the manager was more likely to find inconsistencies in his/her ratings when using the continuous rating scale. 
Throughout the literature the values used in the scale by which specifications are rated has varied. Many different models have been used with a significant majority utilizing the 1-3-9 rating scheme (Park \& Kim, 1998). This scheme was chosen for this method due to its ease of use as well as the apparent acceptance by the research community.

Also, the manager must rate each specification based on the relative importance to the project with respect to all other specifications. This rating scale is based on the percentage of importance when compared to the most valuable specification. The example below uses a simple design with three driving specifications. If $\mathrm{A}$ is the most important specification, it would receive an importance value of 100 . B is also quite important to the design and receives a rating of 90 , because it is 90 percent as important as A. Specification C is also compared to A and only has one quarter of the importance of $\mathrm{A}$, thus $\mathrm{C}$ receives a value of 25 .

\begin{tabular}{|l|c|}
\hline & Importance \\
\hline Specification A & 100 \\
\hline Specification B & 90 \\
\hline Specification C & 25 \\
\hline
\end{tabular}

Figure 3-2: Sample Specification Importance Ratings

As the designers attempt to work together to compromise tradeoffs within a design, it becomes important to understand the relative importance of each specification. If two conflicting specifications within a task are rated based on their relative importance to the overall design, the designers will be able to make more informed decisions when making the necessary compromises. 


\subsection{Decompose Project into Subtasks and Calculate Optimal Groups and Sequence}

For complex parts it is important to break the design problem into subtasks to organize the design. As earlier stated, multiple methods exist by which a design can be decomposed, however this research utilizes a decomposition based on design specifications approach. This approach best allows for the linking of design tasks with specifications that correspond to them and allow for the use of both specification correlations and required talent in determining task sequence and required task groups.

\subsubsection{Specification based Project Decomposition}

This step will draw upon the information gathered in the previous steps; however, the management team is still required to determine the tasks required to complete the design. Initially, the management team will view each specification and determine design tasks to address the specific requirements. Each specification will be assigned to at least one task with the possibility of numerous tasks being required to fully address a single specification. Conversely, a single task can also incorporate multiple design specifications as important considerations while completing the given task.

After each specification has been addressed, it may be required to define further tasks to fully define the project design. While most tasks will have information about interrelationships based on the data acquired about the respective specifications, with the addition of tasks that are not linked to any particular specification, it becomes important to manually input relationship status. This can be done using a chart similar to that presented in figure 3 . 


\subsubsection{Calculate the Task Relationships Matrix (TRM)}

The grouping of design tasks is one of the key value areas of this method. It is first important to calculate task interrelationships utilizing the specification data associated with each task. This is done in the following manner:

First, a new matrix, the specification utilization matrix (SUM) must be created that stores binary information detailing for each task whether it references each specification. As an example, a simple SUM is shown below.

\begin{tabular}{|c|c|c|c|c|c|c|c|c|c|c|}
\hline Tasks & $\mathrm{a} 1$ & $\mathrm{a} 2$ & $\mathrm{a} 3$ & $\mathrm{~b} 1$ & $\mathrm{~b} 2$ & $\mathrm{~b} 3$ & $\mathrm{c} 1$ & $\mathrm{c} 2$ & $\mathrm{c} 3$ \\
\hline $\mathrm{A}$ & 1 & 1 & 1 & 0 & 0 & 0 & 0 & 0 & 0 \\
\cline { 2 - 11 } $\mathrm{B}$ & 0 & 0 & 0 & 1 & 1 & 1 & 0 & 0 & 0 \\
\cline { 2 - 11 } C & 0 & 0 & 0 & 0 & 0 & 0 & 1 & 1 & 1 \\
\hline
\end{tabular}

Figure 3-1 Example of a Specification Utilization Matrix (SUM)

The above table represents a project of three tasks. Each task has three specifications linked to the task. It is not required for each specification to solely belong to one task, but for simplicity this is the case above. This SUM represents which specifications are used in each task and will be labeled matrix S.

The Specification Rating Matrix (SRM) is shown on the following page and will be labeled matrix R. Utilizing these two matrices a third matrix, a task relationships matrix (TRM) will be created for this research that is similar to a DSM except with quantified correlation values between tasks as opposed to specifications. The TRM provides correlation information between tasks that allows for the calculation of the optimal task sequence. This matrix will be labeled $\mathrm{T}$ and is derived and shown below. In this Equation the terms $a, b, i$, and $\mathrm{j}$ correspond to the indices in each respective matrix. 


\begin{tabular}{|c|c|c|c|c|c|c|c|c|c|c|}
\hline Specs & $\mathrm{a} 1$ & $\mathrm{a} 2$ & $\mathrm{a} 3$ & $\mathrm{~b} 1$ & $\mathrm{~b} 2$ & $\mathrm{~b} 3$ & $\mathrm{c} 1$ & $\mathrm{c} 2$ & $\mathrm{c} 3$ \\
\hline a1 & & 0 & 0 & 0 & 0 & 0 & 9 & 0 & 0 \\
\cline { 2 - 11 } a2 & 0 & & 0 & 0 & 0 & 0 & 0 & 9 & 0 \\
\cline { 2 - 11 } a3 & 0 & 0 & & 0 & 0 & 0 & 0 & 0 & 9 \\
\cline { 2 - 10 } b1 & 9 & 0 & 0 & & 0 & 0 & 0 & 0 & 0 \\
\cline { 2 - 10 } b2 & 0 & 9 & 0 & 0 & & 0 & 0 & 0 & 0 \\
\cline { 2 - 10 } b3 & 0 & 0 & 9 & 0 & 0 & & 0 & 0 & 0 \\
\cline { 2 - 10 } c1 & 3 & 0 & 0 & 0 & 0 & 0 & & 0 & 0 \\
c2 & 0 & 3 & 0 & 0 & 0 & 0 & 0 & & 0 \\
\cline { 2 - 10 } c3 & 0 & 0 & 9 & 0 & 0 & 0 & 0 & 0 & \\
\hline
\end{tabular}

Figure 3-2 Example of a Specification Rating Matrix (SRM)

$T_{a b}=$ Value in the TRM matrix at row a column $b$

Rij $=$ Value in the SRM matrix at row $i$ column $j$

Sbj $=$ Value in the SUM matrix at row b column $j$

Sai $=$ Value in the SUM matrix at row a column $i$

$$
T_{a b}=\sum_{i=0}^{n}\left(\sum_{j=0}^{n} R_{i j} S_{b j}\right) S_{a i}
$$

\begin{tabular}{|c|c|c|c|}
\hline \multicolumn{4}{|c|}{ Task Relationships } \\
\hline & A & B & C \\
\hline A & & 0 & 27 \\
B & 27 & & 0 \\
\cline { 2 - 4 } C & 15 & 0 & \\
\hline
\end{tabular}

Figure 3-3 Example of a Task Relationship Matrix (TRM)

With a little Visual inspection it can be determined that from the TRM, the best manner to complete each task would be a serial progression in the order of task B, A, C. However, this tool is best utilized for large complex systems that are not easily identified by inspection. To better illustrate how to move from The Task Relationships Matrix to an optimized sequence the TRM from below has been calculated from a more complex set of specifications and tasks. 


\begin{tabular}{|c|c|c|c|c|c|c|c|c|c|c|}
\hline \multicolumn{11}{|c|}{ Task Relationships } \\
\hline & A & B & C & D & $\mathrm{E}$ & $\mathrm{F}$ & G & $\mathrm{H}$ & 1 & J \\
\hline A & & 6 & 8 & 12 & 11 & 6 & 6 & 2 & 4 & 4 \\
\hline B & 6 & & 28 & 12 & 12 & 9 & 16 & 4 & 3 & 3 \\
\hline C & 8 & 26 & & 21 & 16 & 11 & 24 & 5 & 4 & 4 \\
\hline D & 25 & 12 & 21 & & 14 & 6 & 10 & 3 & 8 & 8 \\
\hline$E$ & 12 & 12 & 16 & 14 & & 11 & 10 & 4 & 8 & 8 \\
\hline $\mathrm{F}$ & 8 & 9 & 11 & 6 & 11 & & 7 & 2 & 6 & 6 \\
\hline G & 8 & 16 & 24 & 10 & 12 & 7 & & 2 & 4 & 4 \\
\hline $\mathrm{H}$ & 4 & 4 & 5 & 3 & 5 & 2 & 2 & & 3 & 3 \\
\hline 1 & 4 & 3 & 4 & 8 & 8 & 6 & 3 & 1 & & 1 \\
\hline J & 4 & 3 & 4 & 8 & 8 & 6 & 3 & 1 & 1 & \\
\hline
\end{tabular}

Figure 3-4 A More Advanced Example of a Task Relationship Matrix (TRM)

As can be seen from the TRM above, when a more complex project is used, the optimal sequence of tasks cannot be determined by inspection. Also, with the large number of interdependencies, each task has some correlation to the others. To calculate the significant task dependencies a threshold value must be determined below which correlations are assumed to be insignificant.

The method utilized for this research is to determine the mean value of each correlation factor as well as the standard deviation. The correlation factors are then compared to the sum of the mean and standard deviation to determine the threshold value. In the above matrix, values above one deviation over the mean are colored yellow while values above two deviations are colored green.

$$
\operatorname{Val}_{\text {sig }}=\text { Mean }+S t d v
$$


Also, while it is recognized that some iteration may still be necessary, it is the goal of this research to minimize this necessity. Notice that on the above matrix, many of the correlations factors with significance have corresponding correlation factors directly across the diagonal that are also significant. For example, Cell $\mathrm{BC}=28$ and $\mathrm{CB}=26$. Because only one task can feasibly be completed first, the larger of the two correlation factors is used while the lower is ignored. Also, often identical correlations factors exist. For example, correlation $\mathrm{CD}=\mathrm{DC}=$ 21. If this is the case, only the correlation factor appearing closest to the top of the matrix will be utilized.

\subsubsection{Group Tasks based on Task Relationships and Calculate Optimal Sequence}

With the TRM calculated, task dependencies are now defined and an optimal sequence can be determined. Using the correlation significance rules from above Figure 3-5 shows preliminary task dependence.

The left column represents a task that should be performed before the corresponding tasks on the left. Note on row B, both $\mathrm{C}$ and $\mathrm{G}$ should be completed after B, but it is not necessarily true that $\mathrm{C}$ needs to be completed before $\mathrm{G}$, although we do see on row $\mathrm{C}$ that this is indeed the case.

From here it is a simple step to go through the tasks one by one starting from the top of the matrix and link the tasks together using a previous/next logic. This idea is utilized in the computer programming discipline when creating linked lists. 


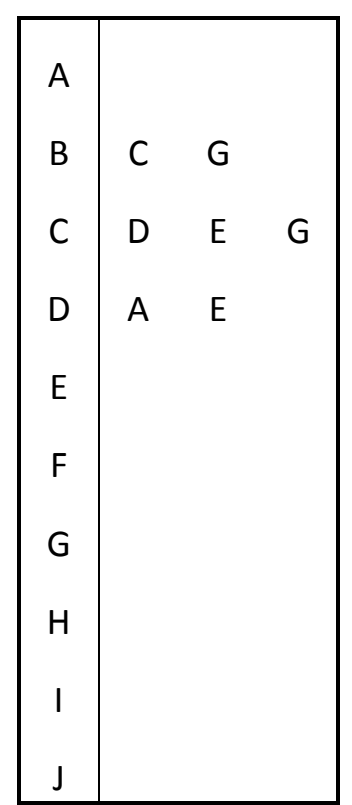

Figure 3-5 Dependencies of tasks in the right column to those on the left

In the above example, starting from the top row, no task would be linked until you reached Row B. Row B would then have the tasks C and G defined as next tasks. C and G would each define $\mathrm{B}$ to be the previous task. Then moving to row $\mathrm{C}, \mathrm{G}$ would be redefined as the next task for C in place of B along with tasks D and E. Finally, on Row D E would be redefined as the next task along with A and the corresponding previous tasks also set. The rest of the rows show no significant dependencies and so they remain independent.

When this is complete, each task will have either no previous task or one previous task. Those with no previous tasks can all be started in parallel by unrelated design groups. There is no limit to the amount of tasks present in the next task list. When there exists multiple tasks in a Next list, this signifies that each of these tasks need to go after the previous task, but can then all be performed in parallel.

Figure 3-6 is the completed task sequence for the example TRM Figure 3-4 from above: 


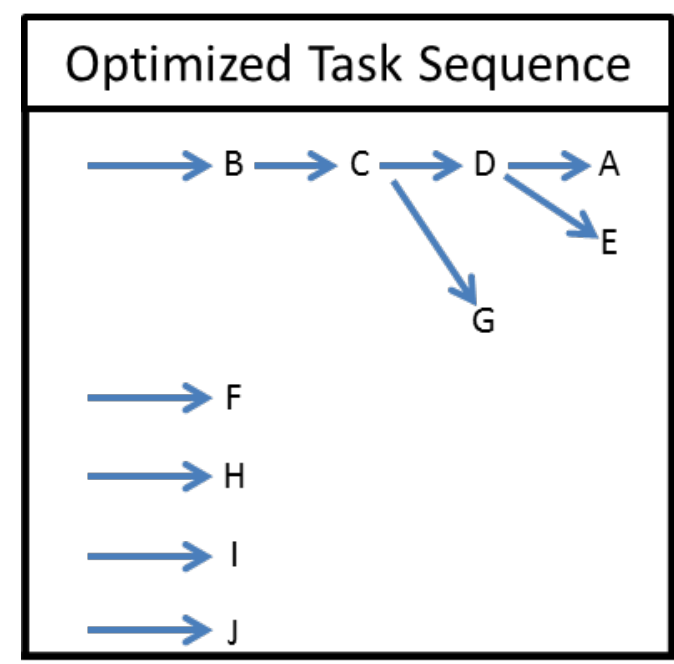

Figure 3-6 Task Sequence Based on the Calculations of Task Dependency

Items that line up vertically can be performed in parallel while those lined up horizontally have a dependent relationship and must be performed in order. Following this task sequence will enable a design team to complete a project more efficiently.

When items can be performed in parallel, separate design groups are created to perform each branch in the task sequence map. In the above example, assuming the tasks are similar duration, it makes sense to break the task into at least two groups. One group will be assigned to perform the task sequence beginning with task B, and other groups can be created and assigned to perform the tasks $\mathrm{F}$ through $\mathrm{J}$.

\subsection{Compose Personnel Groups of Required Talent}

Now that the design tasks have been broken up into groups that can be performed simultaneously, corresponding talent groups are created. These Personnel groups consist of the required talent to perform the tasks in its corresponding task group in the optimal sequence. Often task groups will contain parallel tasks within the task flow that each requires the same 
talent. In these situations, multiple persons representing the specific talent would be required to perform the tasks in the optimal sequence.

\subsubsection{Assign Team Members to each Task}

To create these Personnel groups, the needs of each task within the design group are taken into account. Within the task groups each task will have a required set of individuals representing talent. This talent set will be derived from the needs of the specifications driving each task.

\subsubsection{Group Talent Sets from Each Task into the Talent Group}

Once all tasks within a task group have an associated talent set, those talent sets are joined together in a Talent Group. The Talent group will then have all necessary expertise and decision authority to complete each task in a given task group.

\subsection{Talent Groups Perform Tasks Collaboratively within Multi user Environment}

The preceding steps are the means to arriving at the actual design of the product. At this stage, each talent group has a specified set of tasks to complete as well as a specific sequence in which they should be accomplished. The true value of the method lies in the ability of the design group to perform the tasks in a manner that improves overall design time, and fosters the development of a superior design.

\subsubsection{Each User Participates Simultaneously On Each Task}

The members of the talent group now become the individual users within the multi user CAD environment. Each user will have access to design tools that will allow them to manipulate 
the part file. These changes will be reflected upon the screens of each user within the design environment. While each user has access to these tools and the software presumably will allow for the simultaneous modification of the part file by separate users, the tasks sequence within each design group will predominantly be serial.

Within the serial task list each user required to perform the active task will focus solely on its completion. The task will remain active until all required users determine the task has been completed and they are ready to move to the next phase.

\subsubsection{Communication of Design Rationale}

The great benefit of including all required talent and decision authority into the design session is the ability to communicate the design rationale behind every decision. This communication facilitates the compromise of conflicting design parameters that are each controlled by separate areas of expertise.

Currently designers can meet together to try to determine the best compromise possible when such conflicts arise. However, often the vision of each designer is not conveyed properly to the single user who will ultimately create the design in the CAD system and a series of design iterations must take place to arrive at the final product. With each iterative cycle designers must either address the issue alone without valuable communication from the other decision makers, or other meetings must be scheduled to again bring the designers together.

Furthermore, these meetings between designers are limited to discussing the immediately apparent design challenges and have no way of addressing future difficulties. This leads to multiple separate conflict resolution cycles each of which can become a very time consuming process. 
In contrast, by bringing each of the previous designers together in the same design environment, any conflicts that arise will be addressed immediately. Design iterations will still occur, but they will now be resolved in a single sitting while providing for a more effective environment for compromise and innovation. Another benefit to the overall level of design is that due to the increased ease of design iterations and compromise, designers are less likely to declare a solution 'good enough' before other viable and potentially superior solutions are explored.

\subsubsection{Each User Can Personally Make Changes to the Part}

The main focus of this method is to allow for design rationale to be communicated instantly to all decision makers allowing each to collaboratively determine the optimal solution. It is, however, often difficult to explain ones vision of a part with nothing but verbal communication. By allowing each user to manipulate and modify the part, complex ideas and modeling techniques can be shown visually fostering better communication and understanding.

Current research into the area of multi-tasking also gives a unique perspective. While multi-tasking was initially looked upon as a desirable activity, research now suggests that focusing on a single task and only moving on when that task is completed is a far superior form

of time management (Willingham, 2010). This may be even more applicable to a group of decision makers who are required to make design or approve designs on a number of different projects.

It must be noted that the proposed method requires a shift in thinking for decision makers. Instead of waiting for a design to reach their desk, it is proposed that they are now intimately involved with each of the design phases. This may feel like a waste of resources as a decision maker may feel they are missing the opportunity to make progress on other tasks. 
However, by focusing on one task at a time, the decision maker will be fully aware of the rationale behind each design decision, and will be available to provide instant feedback and approval where necessary. This will foster greater progress toward the completion of the task, and once completed will then free the team up to move on to the next project and apply the same principles. In the end, all tasks will be completed in less time than the conventional method, with a high probability of having arrived at a superior solution. 


\section{IMPLEMENTATION}

This chapter demonstrates the tools created to demonstrate and test the method described in chapter 3. The tools consist of a Specification and Task organizational tool named vManager that acts similar to a product development wizard. The project manager or management group provides a series of inputs and a multi user CAD product design plan is returned as the output.

Also, the multi user tool NX Connect was used in demonstrating and testing the value of working as design groups on a specified task list in a collaborative manner. NX Connect is continually being developed to incorporate more functionality and robustness. The current version utilized for this research was NX Connect 3.0.

The tools created adequately demonstrate the value of the current research, however, both cases fall short of being sufficiently robust to utilize in a commercial setting. The details of how these tools were utilized are presented below along with an overview of additional functionality implemented into the NX Connect software for this research.

\section{1 vManager - Product Development Process Software}

The vManager was created to utilize the power of computing and data storage to facilitate the creation of optimal design groups and task sequence. The software utilizes design Specifications along with the correlation factors between the specifications to facilitate in creating design tasks. Storing and utilizing the correlation information between specifications 
and later calculating and utilizing interrelationship data between the tasks is well suited for computer tools as the information can be extensive and contains many complexities. With a larger and more complex product the amount of information required increases exponentially. By developing a computer software tool to manage the data, we are able to utilize this method in a scalable fashion.

\subsection{Input and Rate Specifications}

The Software consists of two main dialog boxes: the Specification window, and the Tasks window. Each of these windows is also made up of a series of tabs that allows the project manager to step through the tabs in order to input all the necessary information with respect to the design specifications.

The initial tab of the Specifications window shown in Figure 4-1 allows the manager to input the design specifications that will define the product. The manager will then enter the type of criteria that will be used in evaluating the specification, namely, a value, or a specific textual description. Then the acceptable range for the specification value will be input with a target value field also available. In this case, it is understood by the end user that a zero value denotes the lack of a specific target value and any value within the given range is acceptable.

As each Specification is entered and saved into the system, the list located at the bottom of the window is populated. Should the manager wish to review a specification and make a change the specification should be selected from the list and the current information will appear in the input boxes and are subject to editing. 


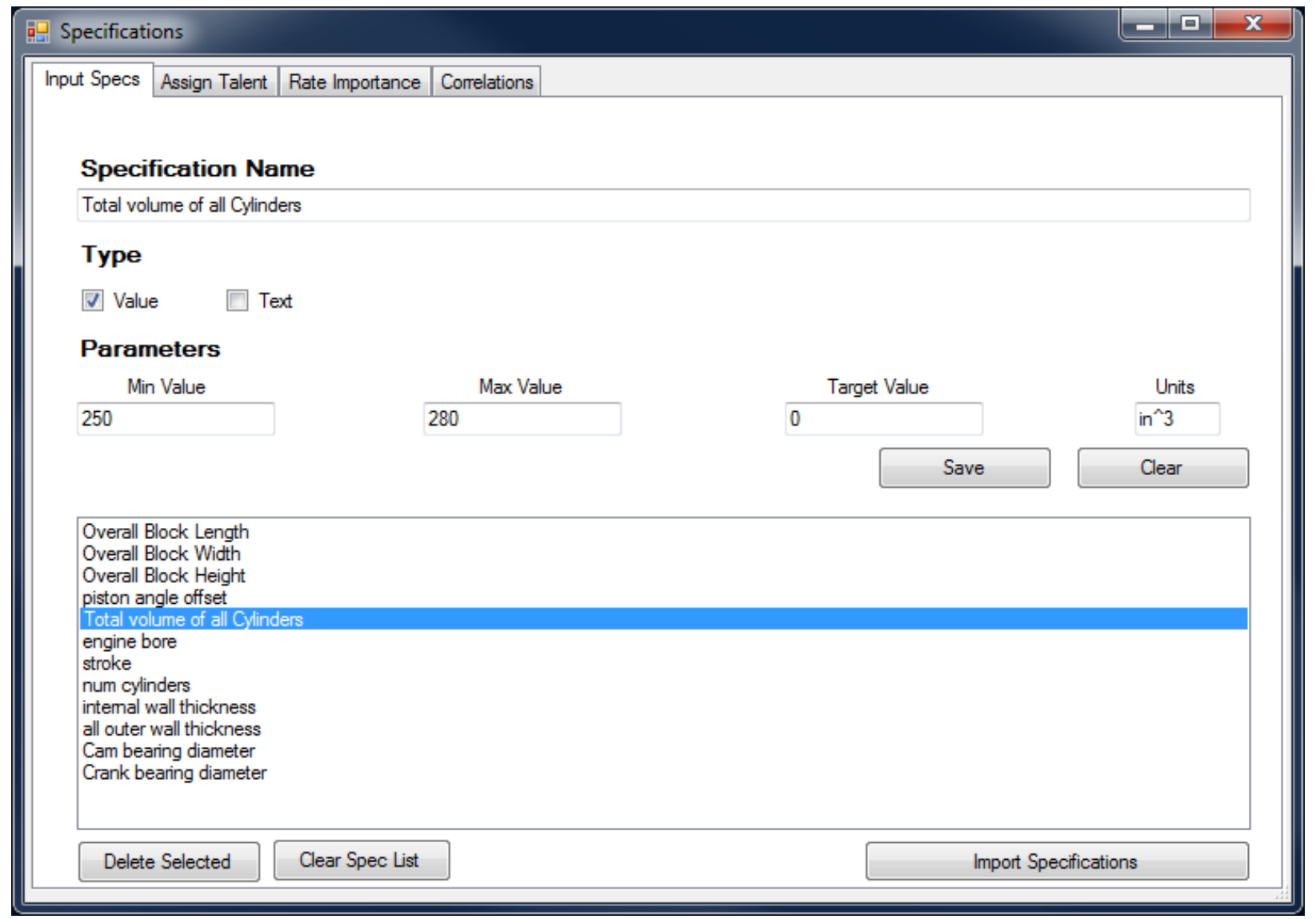

Figure 4-1: vManager Input Specifications Tab 


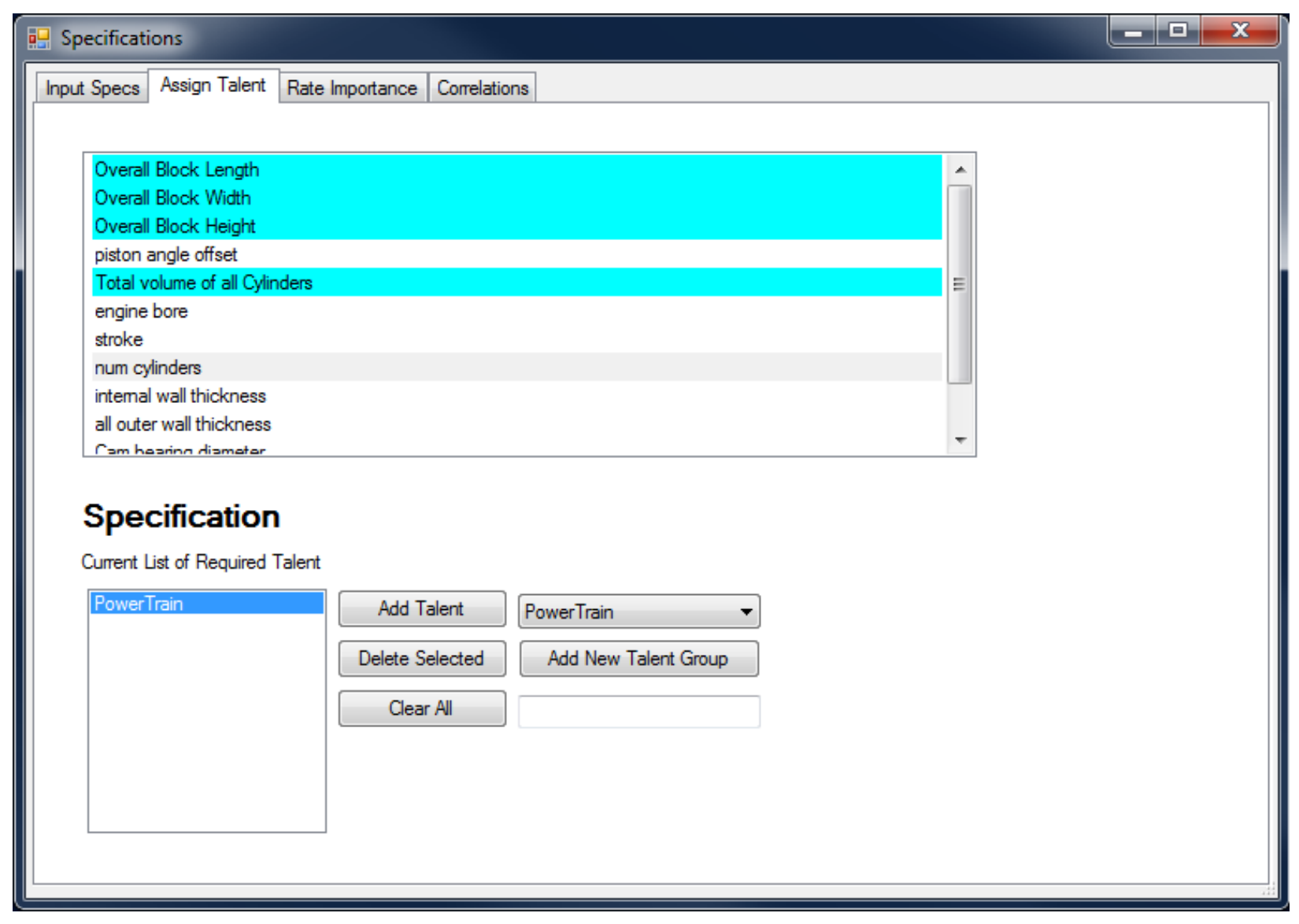

Figure 4-2: vManager Assign Talent Tab

The second tab allows for the manager to select the talent required to fully define and design for a particular specification. The manager selects the specifications from the top list and can utilize the drop down menu to choose which talent group to assign to the specification. If the appropriate talent group does not appear in the drop down list, it can then be added via the bottom textbox and will then be available in the drop down menu for the remainder of the specifications. When the correct talent is selected, the user will select 'Add Talent' and the talent will be saved to that specific specification and will display in the list to the left.

As each specification is assigned a minimum of one talent, the specification in the top list will then be highlighted to indicate that the specification has already been addressed. Should the 
manager wish to remove a talent from a certain specification, the specification is selected, the talent to be remove is selected, and the user presses the 'Delete Selected' button. For best results of the software, each specification should have an assigned talent to ensure the proper personnel is assigned to work on tasks involving this specification.

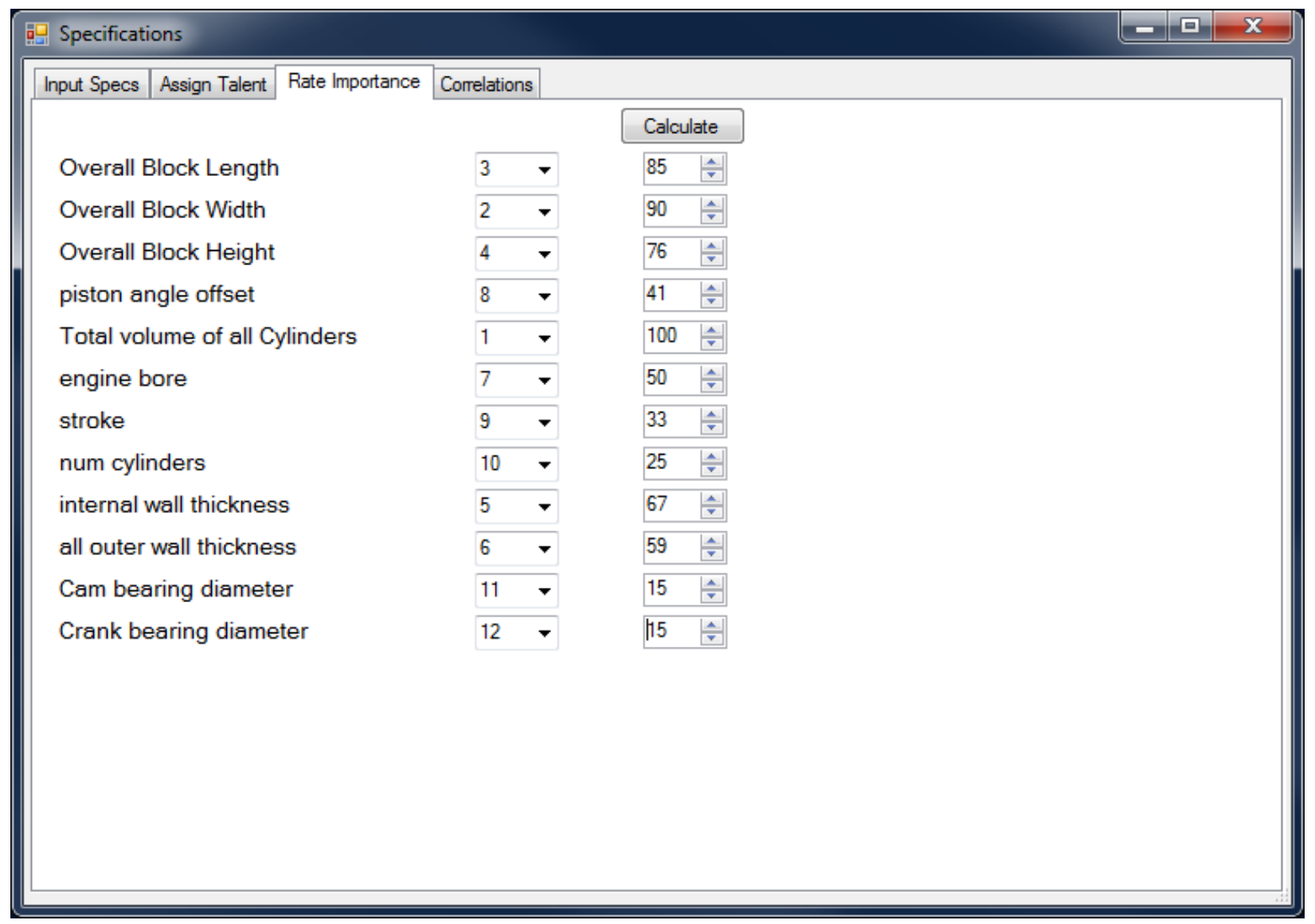

Figure 4-3: vManager Rate Importance Tab

The Rate Importance Tab is utilized to input the relative importance of each task. First all the tasks are ranked by the user according to their sequential importance from 1 through $n$, with $\mathrm{n}=$ to the number of design specifications. The highest ranking specification will then receive a value of 100 while the remaining will receive a linearly calculated value based on the 
specific sequential rating. The user will then have the ability to adjust the values of the remaining specifications with the specifications of lower ranking than the adjusted specification automatically updating to a linear value based on the changed importance value.

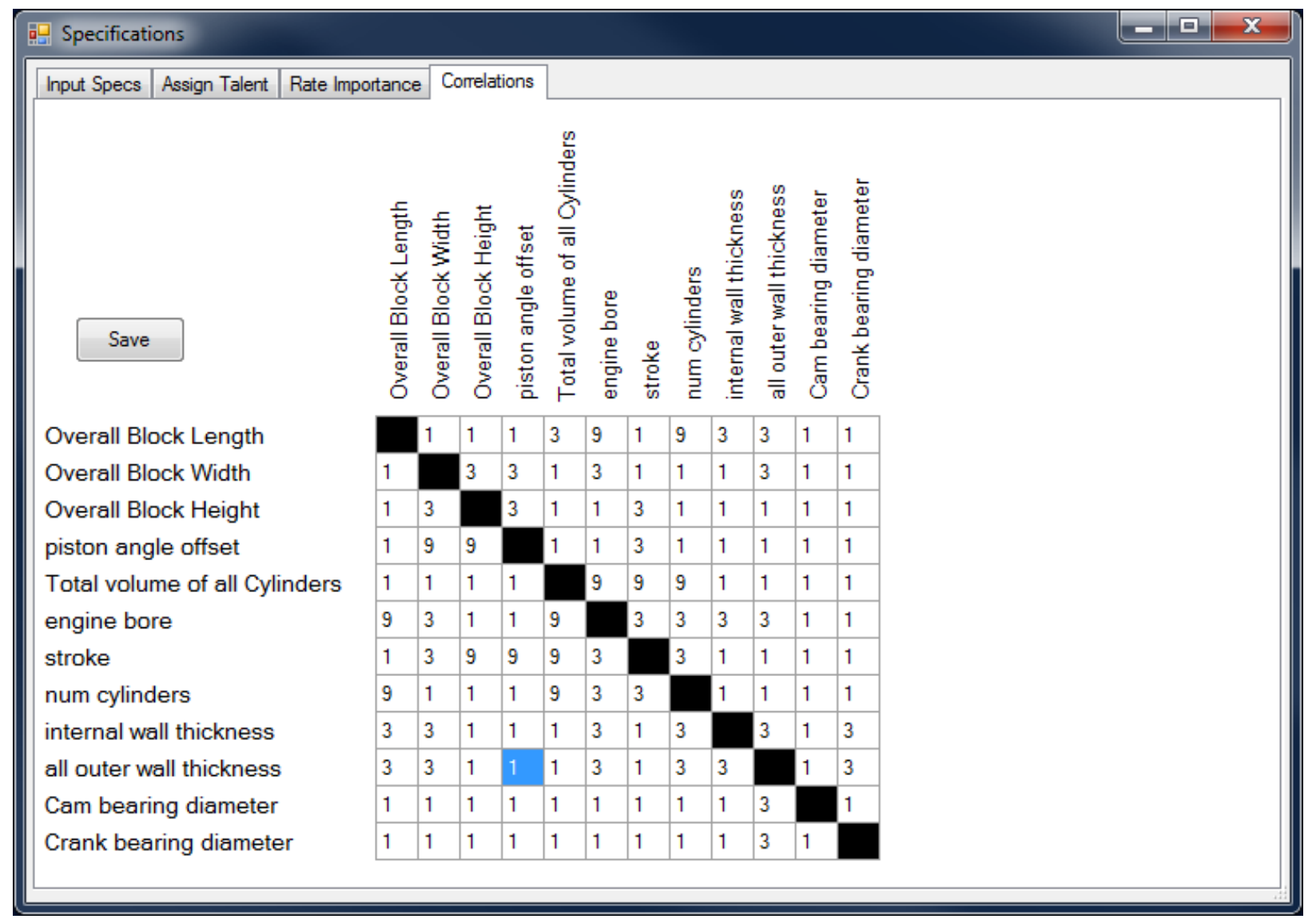

Figure 4-4: vManager Specification Correlations Tab

The final tab of the specifications window utilizes an SRM to allow the manager to define the correlations between each of the specifications. The process to accomplish this is to focus on the first row and input a value in each of the columns along the first row. The values are ascertained by determining how much of an effect on the current specification a change in the value of the specification in the column of interest would have. If the current specification 
would be greatly affected, a value of 9 would be inserted, 3 for moderate affect, and a 1 for little to no affect.

Once each of the specifications has been rated on correlations, importance, and assigned talent, the user will save the design specifications by selecting 'Save' and will close the dialog box.

\subsection{Determine Design Tasks}

The manager is now ready to move on to the phase of determining design tasks. This phase occurs in a separate window dedicated to tasks which also consists of various tabs. The set of design tasks is meant to be determined by utilizing the design specifications and creating tasks that will incorporate design based on these specs. Then task relationship status will be determined by the software with the manager able to override the conclusions of the software if desired. The software will then determine the task groups, optimal sequence of task performance within those groups, and the talent associated with each group.

The project manager will define the tasks to be performed in the first tab of the Tasks window. This tab displays the list of design specifications associated with the project on the right hand side of the window. The manager will begin with the top specification and determine a task or multiple tasks that will address the design features fundamental to the selected specification. The manager will write the name of the design task in the task name textbox, and highlight all of the specifications that play a role in the completion of the task. Press the ' $<<$ Add' button to include these specifications and then select the 'Save Task' button to save the

task. All saved tasks are listed in the lower portion of the window which can be selected and edited at the manager's discretion. 


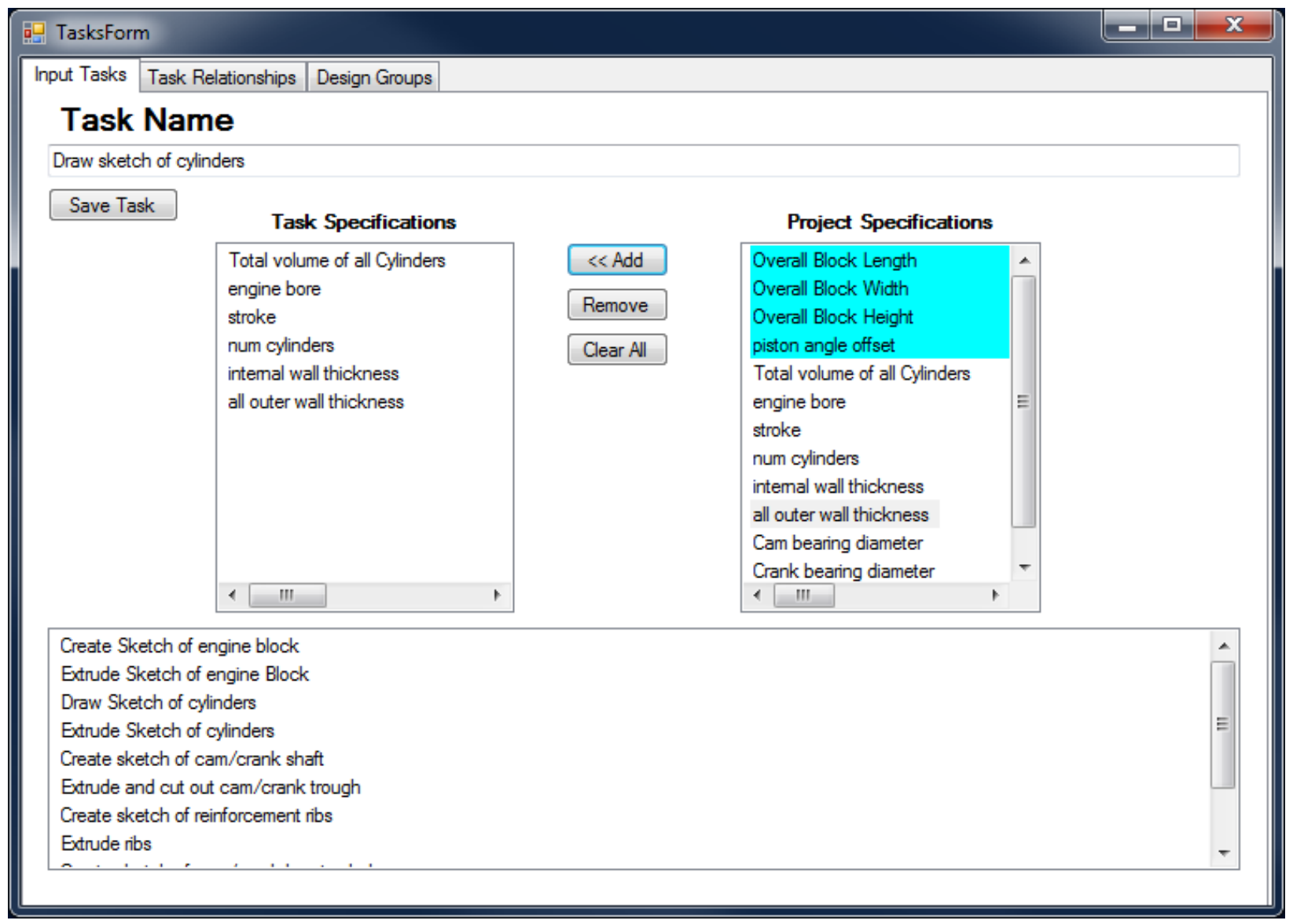

Figure 4-5: vManager Input Tasks Tab

After a specification has been assigned to a task, that specification is highlighted in the list on the right. This provides a visual cue to the project manager to help ensure that all specifications will receive proper attention. Each specification can be used multiple times even after it has already been highlighted. A single task can incorporate multiple different specifications. After all specifications have been addressed, it is often required to enter additional tasks that do not directly reflect upon any specifications, but are required to provide continuity to the design. 


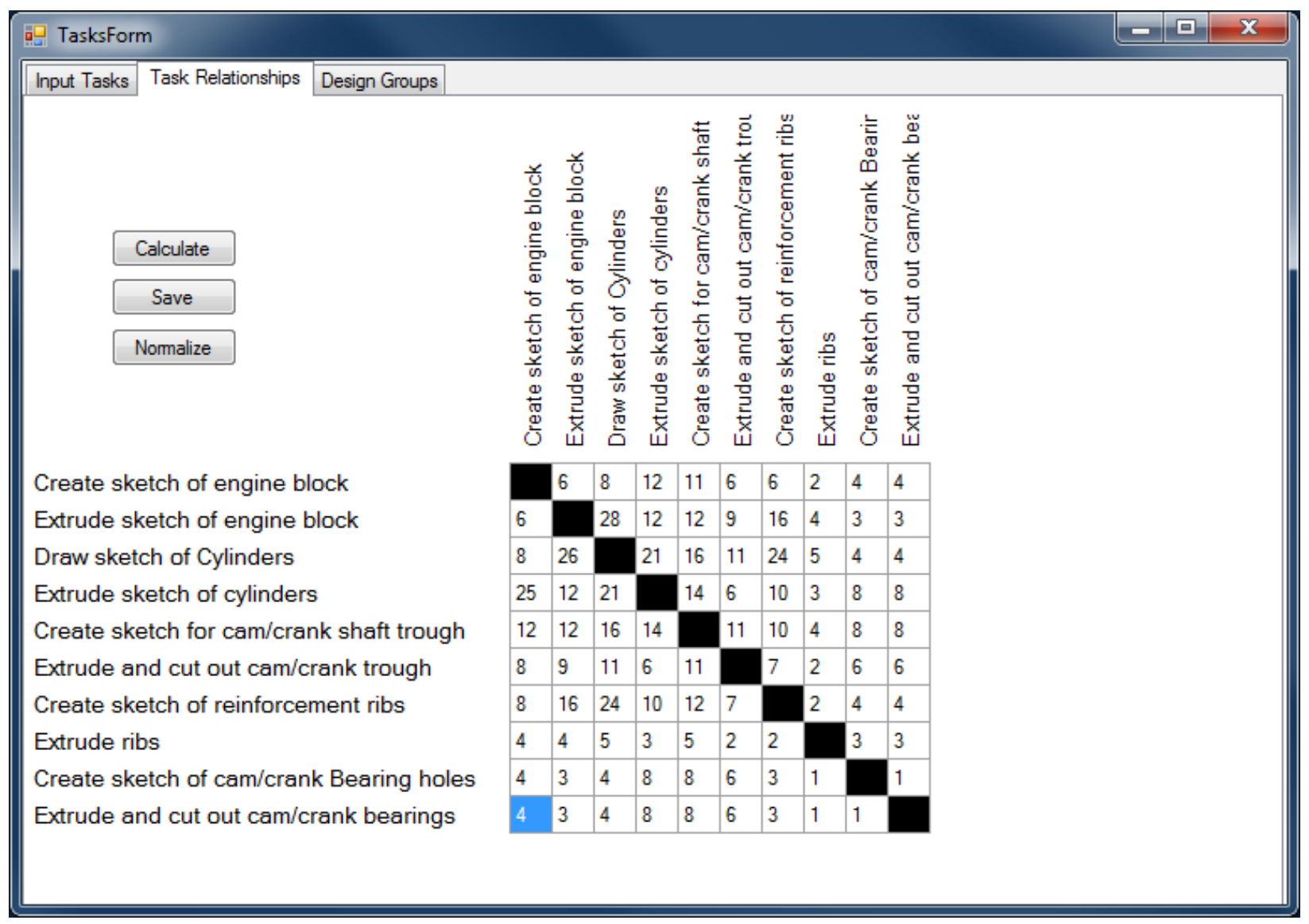

Figure 4-6: vManager Task Relationships Tab

The middle tab utilizes the SRM populated on the last tab of the specifications window as well as the SUM created when tasks were defined and assigned to their respective specifications. These matrices are used to derive the TRM for the project. The TRM represents the dependencies each tasks have on the others within the project and will be automatically generated. This matrix will provide for a visual method to quickly check over the relationship status determined by the software and will allow for modifying the calculated values, as well as provide the opportunity to enter additional dependencies. At this time, any tasks in the system without a corresponding specification will require managerial input for the task dependencies. After the manager has input all required values and determined the software derived values are 
all satisfactory, the input process is accomplished and the software will then calculate the design groups.

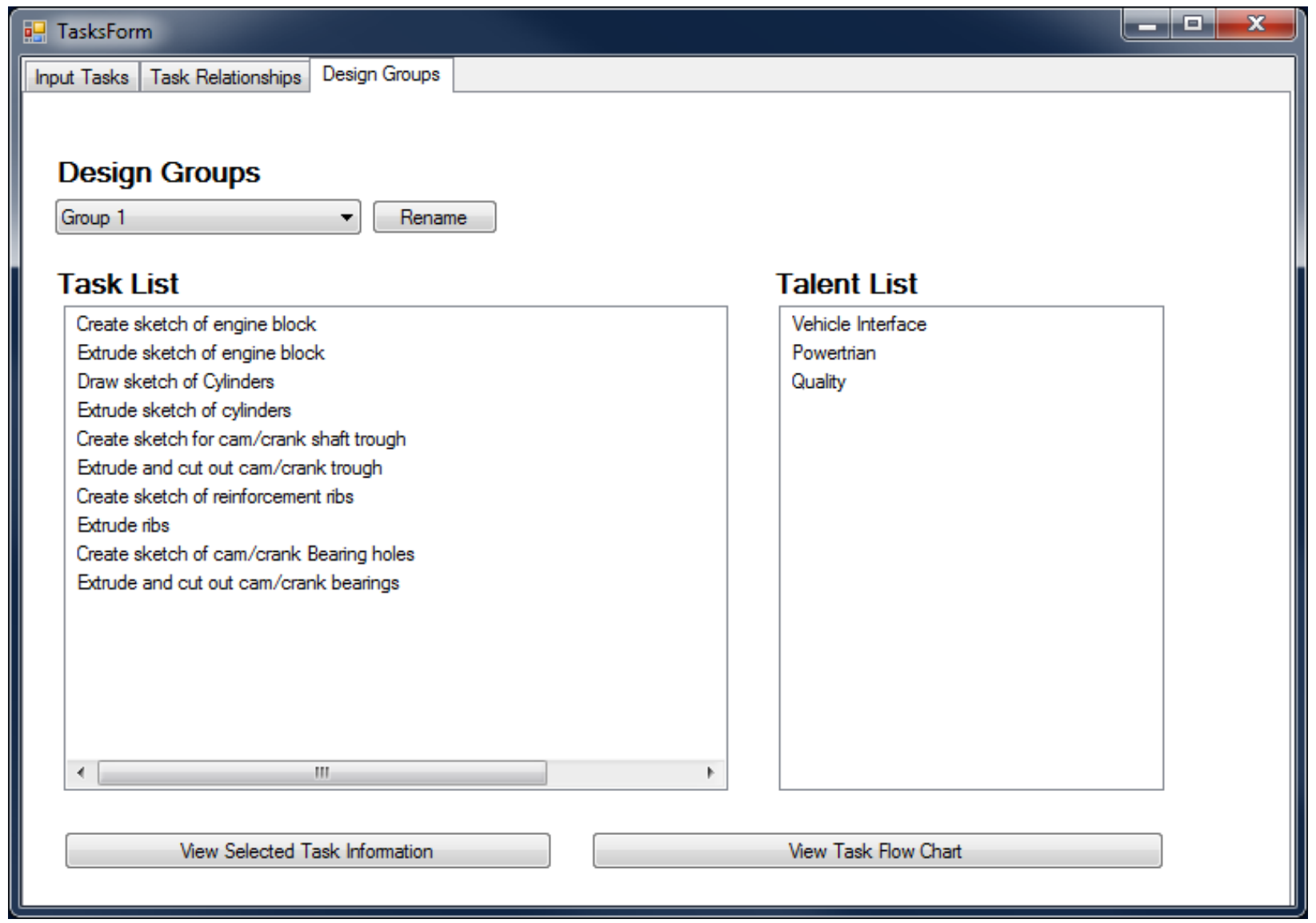

Figure 4-7: vManager Design Groups Tab

The last tab presents the design groups and all the defining information of each group. As seen above, the user can select between the various groups that the software has calculated. When a group is selected, the list of tasks pertaining to the group will populate the Task List box. These tasks are listed in the optimal sequential order to best complete the group of tasks. The Talent List box located on the right side of the window is a list of all the required talent necessary to complete all the tasks in the task list. In this example a minimum of three 
individuals will need to be available during the completion of each of the tasks. Also from this page, the user is able to view the task flow chart to determine the order in which the tasks are to be defined.

Figure 4-8 is the flow chart determined by the vManager software for a specific project. It shows the order by which each task is to be accomplished in a simple easy to understand diagram as well as the required talent for each of the individual tasks. The above example illustrates how it is possible to have parallel tasks even within a task group. These tasks do not necessarily have to be done in parallel but they can be if the required talent is available for each of the design tasks. In the example, task 3 and task 5 can be performed in parallel, however, notice that both task 3 and task 5 require someone from quality to be present during the performance of the task. At this point the manager must determine whether it is more important to include an extra member of quality in the task group, or if the cost of adding an additional team member outweighs that of the potential time savings of performing the tasks in parallel.

Note also that the first few tasks incorporate the car interface talent but no task after two requires this talent. The suggestion of this research is that the user continues to participate in the design process even after their respective talent group has finished its work. This can be very helpful if design difficulties arise further down the line and iterations have to be made that affect tasks that have already been completed. 


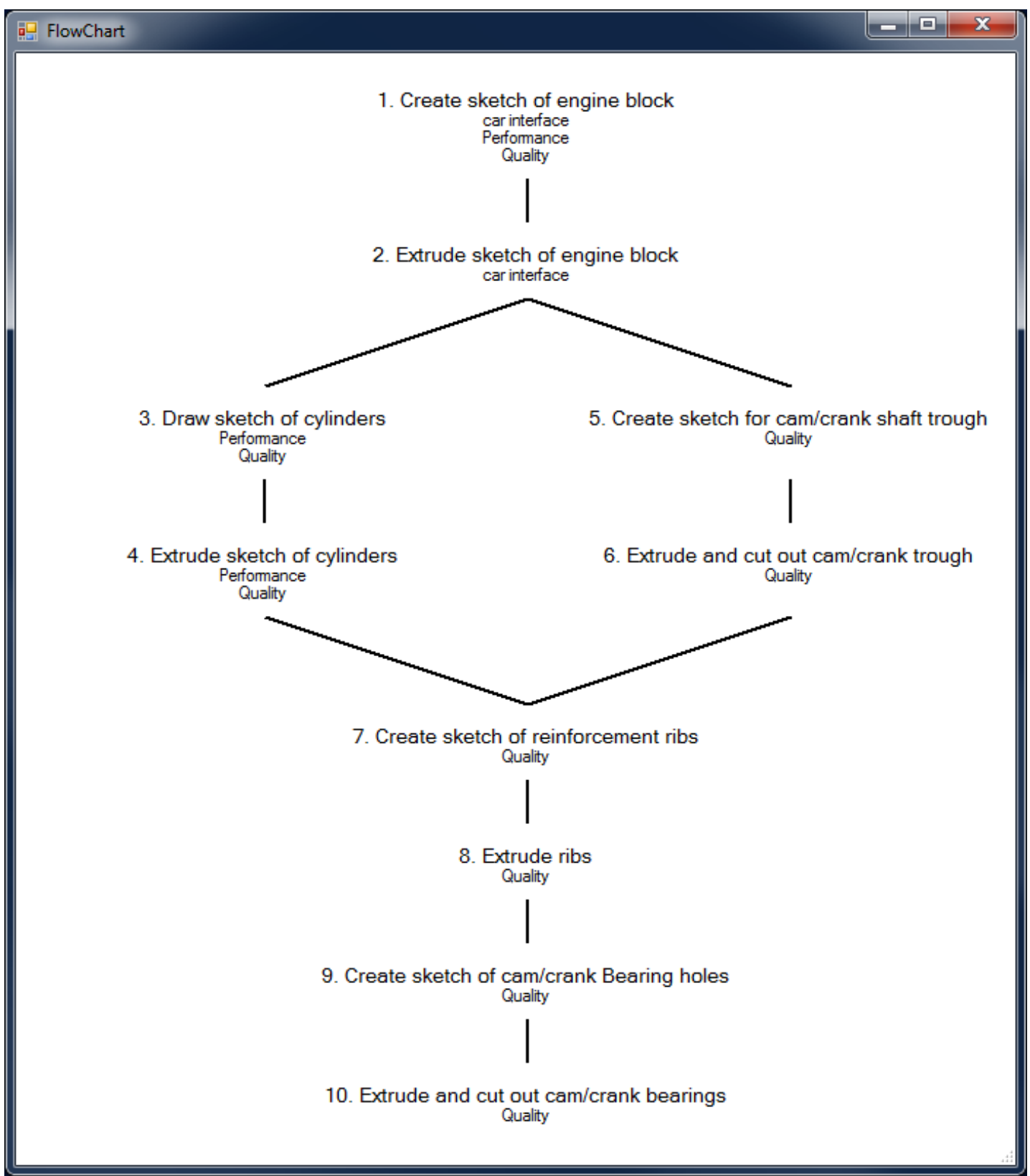

Figure 4-8: vManager Task Flowchart

\subsection{Implementation within NX Connect and Siemens NX 6.0}

The multi user add-on to Siemens NX, NX Connect was utilized to create the multi user environment necessary to implement the final portion of the method. Skype was also utilized to 
set up the verbal communication between each of the users. Successful design sessions have been performed within NX Connect employing the methods described in chapter 3 .

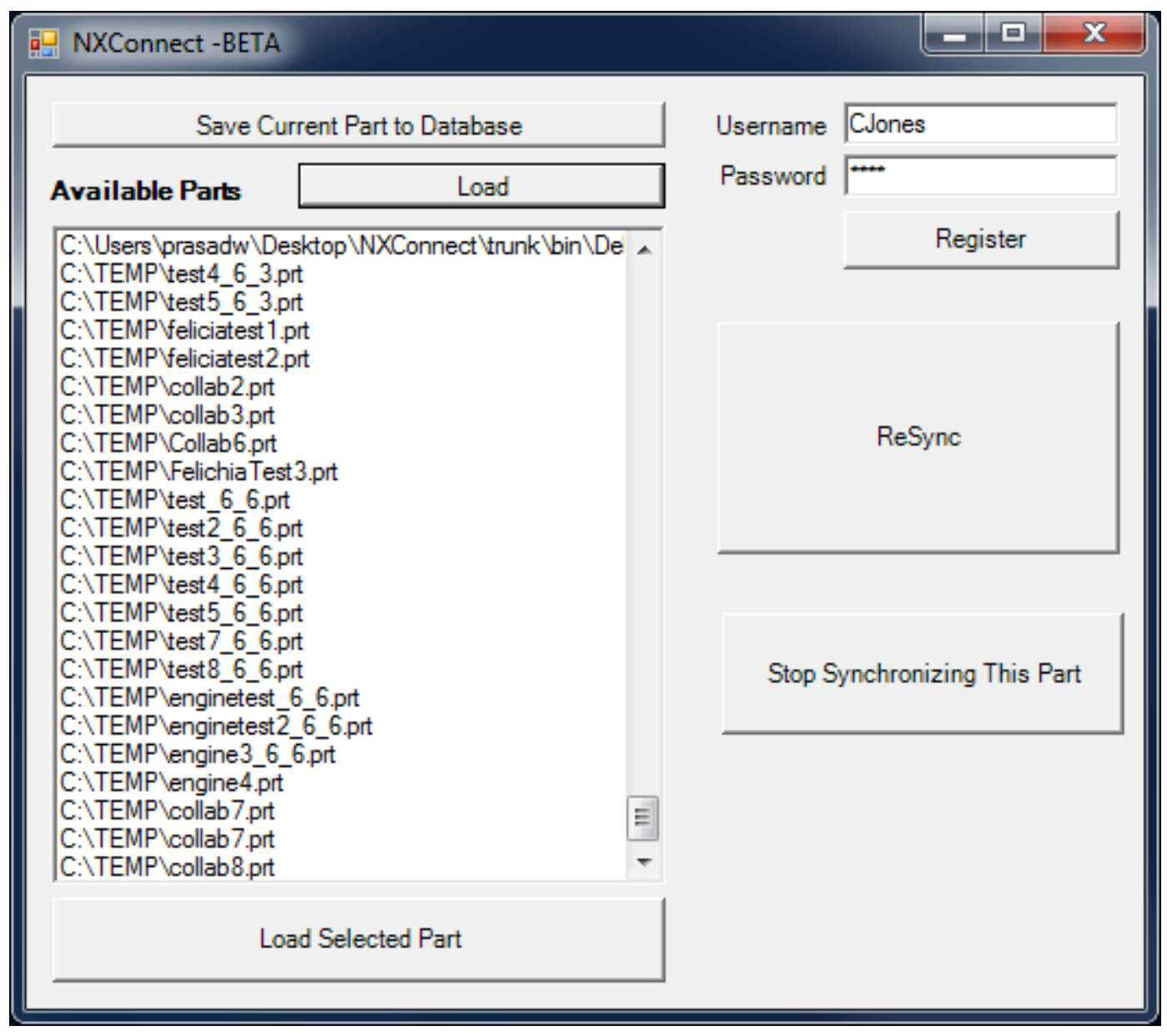

Figure 4-9: NX Connect Interface Screen

NX Connect is run from within the Siemens NX 6.0 software. The above image shows the interface screen where the user can either select one of the existing parts in the database, or can save the current part they have active in the NX session to the database. By saving the part to the database, the part will then become available for other users to open and work on collaboratively. 


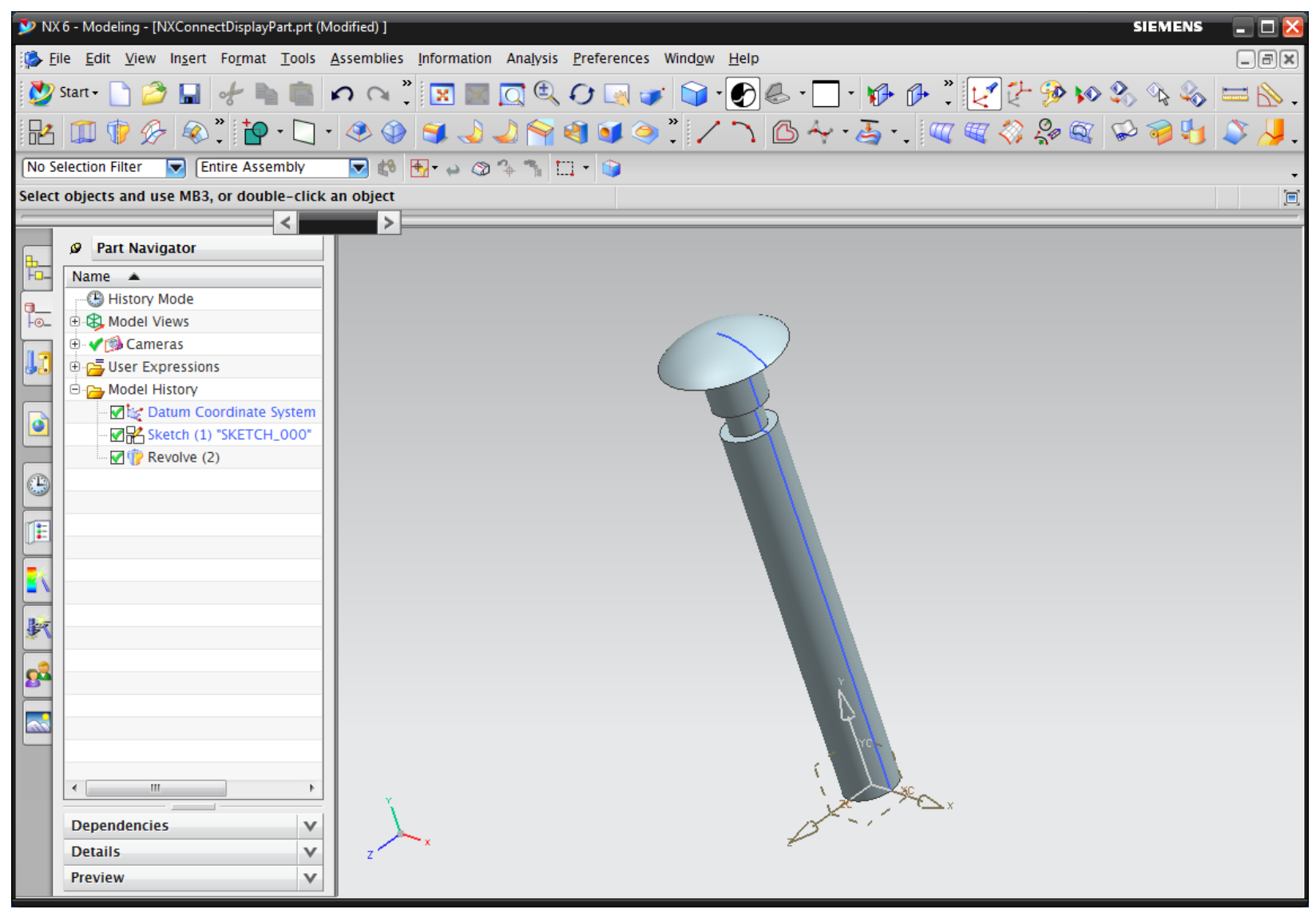

Figure 4-10: NX 6.0 Running NX Connect

\subsection{Increased NX Connect Functionality}

When NX Connect was first developed by Ryskamp (Red, Holyoak, Jensen, Marshall, Ryskamp, \& Xu, 2009) in 2009 it contained functionality that was limited to simple sketches made up of lines and curves, Extrusions, and Boolean operations applied to the extrusions. The software also lacked a great deal of robustness and suffered from many unexplained glitches.

For this proposed research it was important to improve NX Connect to a point where multiple users could truly enter into the design session and design a part together. Much work 
went into the effort of improving NX Connect and while it still lacks many long hours of work, it is now a more valuable tool for future researchers.

\subsection{Developing NX Connect}

Largely related to the architecture and the storage system, the challenge of creating more functionality for NX Connect is that each separate NX function has to be implemented individually. Also, NX Connect is built exclusively using the NX .Net API to interface with the commercial CAD system. This also presents hurdles when trying to develop tools the software was not initially designed to perform or support.

Additional functions that have been incorporated into the NX Connect software as part of this research are the following:

- Revolutions about a datum axis

- The ability to sketch upon surfaces as opposed to planes only

- Mirroring of sketches

- Extrusions of mirrored sketches

- The ability to re-sync a part with the database

- Deletion of features

- General bug fixes and an improved model for organizing the feature history id

With the improved functionality, NX Connect is much more capable of performing the role of a multi user design tool. NX Connect provides valuable insights into how multi users interact and work within a CAD environment; however several limitations still exist in providing for a complete multi user experience. 
A possible improvement that can feasibly be implemented in the near future would be the addition of screen sharing capabilities. This would further allow each user to understand the rationale behind each designer's decisions as they could see them performing each action instead of simply waiting for the finished feature to update onto their own screen.

Also, one of the greatest limitations still present within NX Connect is the sketching environment. At this time the sketching environment is still a single user process with no ability to view the sketches being created on the screens of other users. This is a significant problem due to sketches being an area where the most complex geometry is commonly defined. This is the case in the previous example shown in Figure 4-8 where the initial sketch is the only task within the task group that incorporated the expertise of all required users in the design session. The before mentioned screen sharing feature would help significantly to at the least allow each user to view and provide meaningful input within sketches. For the current research, all communication about sketches is performed verbally, the modeler being chosen based on the expertise that most closely matches the task to be performed. 


\section{RESULTS}

To determine the value of the proposed method an analysis was performed using the tools discussed in Chapter 4 where possible. This analysis is a comparison between methods currently employed in industry, and the method proposed by this research. However, as previously stated, the proposed methods are intended to be used with complex parts consisting of a great number of design specifications encompassing multiple different talent areas within the company. However, due to a lack of resources, qualified testers, software robustness, and time it was not feasible to perform a large scale test on a complex part. Therefore, to show the value of the research, a simulated test was developed that represents a design on a more complex level. The simulated test provides opportunity to test important aspects of the proposed method; however, the low level of complexity limits the ability to fully incorporate all portions of this research.

\subsection{Developing the Test}

The value of the method lies in its ability to bring multiple designers together in the same environment each from a different talent background, with a clear direction to complete the design. Each user is to take full or partial ownership of the design specifications that correlate to their respective talent. Due to the large volume of design specifications it is not realistic for a single design engineer to be expected to know and understand all defining specifications. 
The test was made up of two separate trials to be run with multiple test groups. The first trial measured time and success of a design team to model an engine block in a serial manner. This trial was developed to approximate an iterative cycle that occurs commonly in today's engineering world. The second trial places the users in a multiuser collaborative environment while performing the same task.

The target specifications for the engine block were defined and talent was assigned to each specification as discussed in section 3.2. The design project was decomposed into subtasks (section 3.3.1) based on the provided specifications, and prior knowledge of simplified engine block modeling. To provide a design simple enough to be completed within a reasonable amount of time it was necessary to reduce the level of complexity to the point that the steps to accomplish design were straightforward. This limited the effectiveness of the portion of this research found in section 3.3.3, to provide an optimized task sequence. Hence, calculations of specification and task dependencies to arrive at an optimized task sequence were not included in the test. An identical sequence of tasks was provided to groups performing both trial one and trial two, making this a common element between the two trials.

By basing the initial project definition on the proposed research, the design of the engine block was laid out in a series of simple tasks that would fully define the project and took into account each of the required design specifications. Each task was linked to design specifications which in turn provided information on the required talent group to perform the task.

In the trials, each user represented a member of a design group with a specific talent or expertise. For each trial, three users were selected and each received a different portion of the design specifications for the design of the engine block. The specifications they received corresponded to a specific talent simulating the idea that each user had expertise in a certain area 
of the design. While an identical task sequence was provided for both trials, information linking design tasks to the corresponding talent personnel was made available to only those of trial two, the multiuser collaborative group.

In the first trial, the users were instructed not to converse with each other about their respective specifications. One user would first design the engine block using as guiding values only those specification that have been provided. After the first user is finished, the second user would then receive the part file and proceed to modify the model until the specifications of User 2 are satisfied. After User 2 finishes, User 3 would then take over and attempt to achieve the supplied specification by modifying the part. By this time, the part model has changed significantly and is sent back to designer 1 in an iterative process. This iteration cycle continues until the values of each specification are within its acceptable range, or the allotted time for performing the test has expired.

This first trial simulates designers or stakeholders who, without the ability to work together within a CAD system, are only able to design specifically for the area about which they have complete knowledge and then pass the design down the line.

In contrast, the second trial follows the pattern specified in section 3.5 in which each of the required talent or stakeholders are placed in a collaborative design environment. They have the same task list, but now each designer has a clear idea of his/her role in the design, and the roles of the other members of the design session. The users were placed on separate machines with a Skype conference call set up to allow communication between the users and were instructed to ensure that they worked together to accomplish each task. The users were not to move on to another design task until each of them agreed that the previous task was completed to the satisfaction of all specifications. By performing this portion of the test, each user again 
represented individuals from different areas of the company each having unique talents and understanding of the product to be built. However, they now had access to multi user CAD tools, VOIP software, as well as a more thorough understanding of each of their roles in completing the tasks that made up the task list.

As a result of these trials, a comparison can be made between the effectiveness of an iterative design process between three stakeholders each working individually on the design, and the effectiveness of representatives of the same three stakeholders placed in a multi user collaborative environment.

\subsection{Testing Observations}

In the first trial, it was noted that many users had considerably different visions of what the final product should become. This seemed to present significant challenges as the design would change hands between them. In the second trial, there was no noticeable conflict in final vision, likely due to a natural progression of the design through good communication and collaboration.

In the multi user trial each user had equal ability to perform the various modeling features and much of the time the modeling responsibility was handed off to the team member whose talent was associated with the current design task. During the design process itself, communication was strongly encouraged, and was utilized in a very effective manner. As was expected, each user was able to communicate information effectively about design specifications and requirements, but abundant communication also occurred regarding improved design and modeling techniques. 


\subsection{Testing Results}

The test subjects used to perform the tests were on a time constraint and multiple test groups were not able to finish the tests in the given amount of time. Because of this, the time of modeling was collected, as well as a modeling score. The modeling score was calculated on a scale of $1-10$ with one point being awarded for each of the specifications that was successfully met. A deduction factor was also used to penalize teams for incomplete or inconsistent models. Data was collected from the various test runs and is presented in Tables 5-1 and 5-2.

Table 5-1: Single User - Iterative Design Test Results

\begin{tabular}{|c|c|c|c|}
\hline \multicolumn{4}{|r|}{ Single Users - Iterative Design } \\
\hline Case & Time (min) & Total & Notes \\
\hline 1 & 48.40 & 7 & $\begin{array}{l}\text { Model was completed, but specifications for piston angle offset, } \\
\text { external wall thickness, and cam shaft bearing diameter fell out of } \\
\text { scope }\end{array}$ \\
\hline 2 & 34.12 & 7 & $\begin{array}{l}\text { Model was completed, but specifications for total cylinder volume, } \\
\text { cam shaft bearing diameter and crank shaft bearing diameter fell } \\
\text { out of scope }\end{array}$ \\
\hline 3 & 47.40 & 5 & $\begin{array}{l}\text { Model blew up while users were trying to fix the part to reflect } \\
\text { their specifications }\end{array}$ \\
\hline 4 & 46.88 & 9 & $\begin{array}{l}\text { Complete part but some surfaces, and extrusions are not clean } \\
\text { and do not extend all the way through }\end{array}$ \\
\hline 5 & 37.07 & 7 & $\begin{array}{l}\text { Part is non-symmetrical and the pistons do not extrude through } \\
\text { the entire block }\end{array}$ \\
\hline 6 & 32.37 & 7 & $\begin{array}{l}\text { Interior reinforcing ridges were not fully created, external wall } \\
\text { thickness specification not met, and cylinder locations were } \\
\text { inconsistent. }\end{array}$ \\
\hline & 41.04 & 7.17 & AVG. \\
\hline
\end{tabular}


Table 5-2: Multi User Collaborative Design Test Results

\begin{tabular}{|c|c|c|l|}
\hline \multicolumn{4}{|c|}{ Multi User - Collaborative Design } \\
\hline Case & Time (min) & Total & \multicolumn{1}{|c|}{ Notes } \\
\hline 1 & 35.77 & 9 & Completed part, extruded reinforcing ridges not uniting with body \\
2 & 30.70 & 10 & Complete part, within all specifications \\
3 & 26.30 & 10 & Complete part, within all specifications \\
\hline & $\mathbf{3 0 . 9 2}$ & $\mathbf{9 . 6 7}$ & AVG. \\
\hline
\end{tabular}

More data was able to be collected for the initial single user case because as the three individual designers rotated around, they were able to work on three separate cases each of which was unique due to the different starting points. For the multi user case however, each case represents 3 separate users signifying that in actuality, more test subjects were utilized in testing the multi user method than the former.

The initial single user runs varied a great deal. The number and extent of modifications required between users varied greatly from one case to the other. Some of the designs required enormous modifications to meet the design requirements of subsequent designers in the iterative cycle, while in others the initial design required only slight modifications to meet the specifications of the later designers. 


\section{Single User Case Examples}

\section{Case 2}

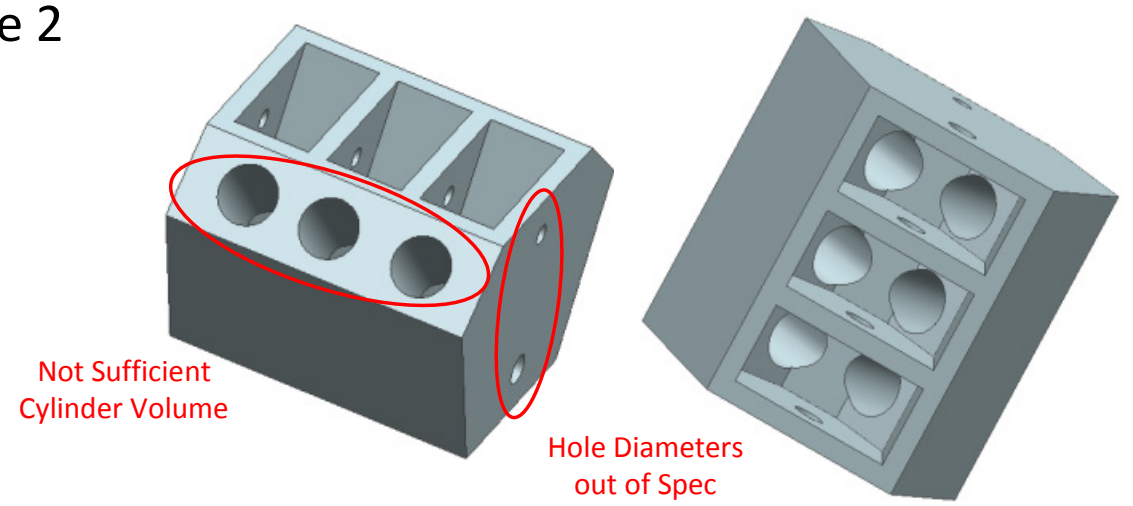

\section{Case 3}
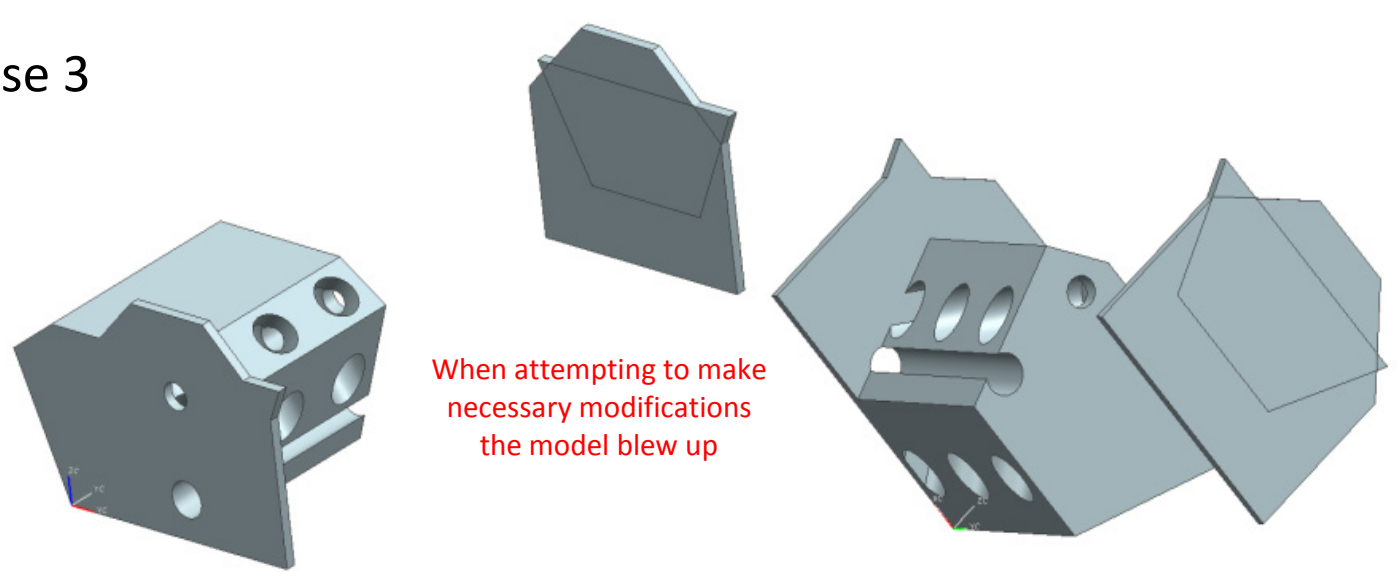

Figure 5-1: Single User Design Test Sample Images

Above are sample figures from the single user iterative design cases. Case 2 shows a completed design, however upon inspection the cylinders are not the correct geometry to produce the required engine volume, and the holes for the cam and crankshaft bearings are both out of the acceptable specification range. Case 3 shows the result of users attempting to make major modifications to a design to meet their respective specifications. The design had been created in a way that when the changes were made the model was no longer usable. 


\section{Multiuser Case Examples}

\section{Case 2}

\section{Case 3}
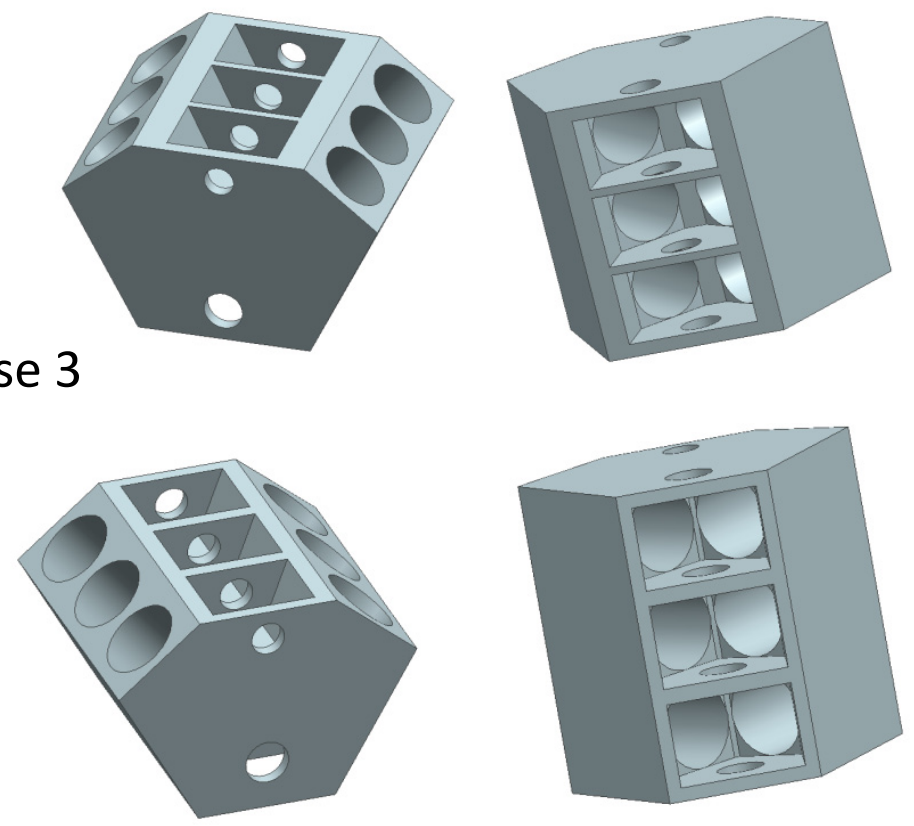

Figure 5-2: Multi User Collaborative Design Test Sample Images

The multi user test produced much better and more consistent design scores, and the completion times were significantly better than those of the groups working serially. The test users were able to complete the design without the need to return and make major modifications later due to improved organization, better defined roles between the designers, and the ability to communicate the design needs during each stage of the design process.

The sample multi user cases show that the engines were completed and upon initial inspection, they passed the eye test. Also, upon further examination, the specifications are met completely in all cases. 


\section{CONCLUSIONS \& SUGGESTIONS FOR FUTURE WORK}

In the world today, engineers are exploring more complex designs than ever before. These designs require a great deal of knowledge and expertise in a vast amount of varying disciplines. Collaboration is a basic requirement to find success as engineering companies attempt to keep pace with the rest of industry. Current engineering software tools have however been a hindrance to the efforts of collaboration as they are architecturally limited to single user design. Recent innovations to engineering tools within academia have provided the basis for future collaboration within these engineering tools by providing limited multi user access to widely utilized CAx applications.

The purpose of this thesis was to present a method that would allow design companies to take advantage of the benefits provided by multi user CAD software. This is in response to questions regarding the efficacy of utilizing multiple designers in the design space due to increased probability of design conflicts. By following the method prescribed in this text, tests have shown that multi user teams have superior final designs and have completed those designs in significantly less time.

Through an analysis of current shortcomings and bottlenecks in the design process it was determined that a large portion of design time is utilized by iteration cycles. These iteration cycles tend to be frustratingly slow as designs are passed from one decision maker to another. Each time the design is handed off time elapses as the new designer waits for schedule openings 
to address the design, as well as an initial design review to familiarize him/herself with the current details. With every modification made by a designer in the iteration cycle, the design has to be reviewed again by all other required decision makers.

The use of multi user software allows for an effective solution to the problem of long iteration cycles. This research proposes a method in which all required decision makers are determined and brought together in the same design session. The design session will consist of a list of tasks with high interdependence requiring the input of each of the decision makers to fully complete the task list. These task lists are generated from information about the project as defined by the project manager or management team with the tasks optimally sequenced based on task dependency.

The value of the presented method lies in the determination of each necessary individual required for approval to move forward with design, and placing each of those individuals in a collaborative environment with clear direction to complete the design. Each of the designers is uniquely qualified in their respective areas to make decisions and provide input to help achieve the target specifications. Where the specifications seem to conflict and compromises must be made, each designer has had the opportunity to observe the rationale of the design and can better weigh the importance of specifications pertaining to their area of expertise. Instead of going through a long iterative process, designers can communicate directly to each other and arrive at the required compromise in a minimal amount of time. This is in stark contrast to the idea of figuratively "throwing the design over the wall" to the designer from a different discipline and background and waiting for it to return.

Multi user software is a necessity for this method to function most efficiently. By providing all decision makers an atmosphere of collaboration, and a medium by which they can 
visually show their design ideas, communication will drastically improve, and as a byproduct, finished designs will be far superior.

The testing performed on this method has supported this claim. In each of the test cases, a multi user design session outperformed a multiple single user iterative process. The multi user designs were superior in quality, as well as the overall time to arrive at the finished design.

In conclusion, this research provides a valuable method to organize a design project into sequentially optimized task lists, complete with the required set of personnel to address the issues and conflicts that may arise during the design process. When these tasks are performed with each designer present and participating in a multi user setting the results are far superior to those of the traditional single user iterative design process.

\subsection{Recommendations}

The value of utilizing the vManager tool and the multi user design method have been shown through this research, however the implementation of the method lacks robustness. Primarily, the tool NX Connect, while significantly improved for this research still lacks much of the capability that is available within the NX CAD software. Continued development of the NX Connect tool will allow for more seamless multi user design sessions especially when parallel design groups perform tasks concurrently with another. Also, NX Connect is limited to the NX 6.0 CAD system and is incompatible with all other CAx tools. The limited scope of the testing was due in large part to the limitations of the multi user CAD software available. Future work is suggested into continuing the development of NX Connect as well as developing and utilizing similar tools for various other CAD software packages.

As previously mentioned, the testing that was performed for this research was very limited. Resources were not available to perform a comprehensive test of the method presented. 
It is suggested that a minimum of two further tests should be performed. The first test is to be a significantly more complex part, which would require large sets of specifications and of required talent to complete the design. The second would be a test utilizing a complex assembly that could be designed and assembled in a multi user environment. These tests should fully incorporate the project management software vManager to define the task sequence and talent required.

The vManager software also possesses significant limitations. Presently the software is not capable of holding large amounts of data as would be required for fully functioning management software. Also, the vManager has no way of determining if a product has been fully defined. This could present significant problems if the product manager fails to fully define intermediate tasks to preserve task continuity while performing the design. Under these circumstances, the software would produce a task flow chart that would not be a valid design path. Future work is suggested into developing a method to determine level of definition of a given project. 


\section{REFERENCES}

Google Docs. (2011, May 22). Retrieved May 27, 2011, from Wikipedia: http://en.wikipedia.org/wiki/Google_Docs

Byrch, F. (2010, August 27). Cisco. Retrieved May 23, 2011, from Cisco Communities: https://communities.cisco.com/videos/5990

Chanron, V., \& Lewis, K. (2003). A Study Of Convergence In Decentralized Design. ASME Design Engineering Technical Conferences. Chicago, Illinois: ASME.

Feng, L., \& Chen, L. (2006, September). Achieving Online Coordination in Real-Time Collaborative Assembly Modeling: A Supervisory Control Approach. 6(3), 252-263.

Ford, D. N., \& Sterman, J. D. (2003, September). Overcoming the 90\% Syndrome: Iteration Management in Concurrent Development Projects. Concurrent Engineering: Research and Applications, 11(3).

Fuh, J., \& Li, W. (2005). Advances in collaborative CAD: the-state-of-the-art. Camputer-Aided Design, 571-581.

Hauser, J. R., \& Clausing, D. (1988, May-June). The House of Quality. Harcard Business Review.

Imai, K., Nonaka, I., \& Takeuchi, H. (1986, January-February). The new new product development game. pp. 137-146.

Kao, Y.-C., \& Lin, G. C. (1998). Development of a collaborative CAD/CAM system. Robotics and Computer-Integrated Manufacturing, 55-68.

Li, W., Lu, W., Fuh, J., \& Wong, Y. (2005). Collaborative computer-aided design - research and development status. Computer-Aided Design, 37(9), 931-940.

Liker, J. K., Sobek II, D. K., Ward, A. C., \& Cristiano, J. J. (1996). Involving Suppliers in Product Development in the United States; and Japan: Evidence for Set-Based Concurrent Engineering. IEEE Transactions on Engineering Management, 165-178.

Lock, D. (2007). Project Management (9th ed.). Burlington: Gower Publishing Company. 
Lohr, S., \& Helft, M. (2007, December 16). Google Gets Ready to Rumble With Microsoft. Retrieved May 27, 2011, from The New York Times: http://www.nytimes.com/2007/12/16/technology/16goog.html?_r=1\&oref=slogin\&pagew anted $=$ all

Lowe, T., \& Lowe, R. (2011). QFD Tutorial. Retrieved May 30, 2011, from Webducate: Innovative Solutions for e-learning: http://www.webducate.net/qfd-tutorial/

Lu, Y., Cai, J., Burkett, W., \& Udwadia, F. (2000). A methodology for Collaborative Design Process and Conflict Analysis. CIRP Annals - Manufacturing Technology, 49(1), 69-73.

Marston, M., \& Mistree, F. (2000). GAME-BASED DESIGN: A GAME THEORETIC EXTENSION TO DECISION-BASED DESIGN. International Conference on Design Theory and Methodology. Baltimore, Maryland.

Mishra, P., Varshney, A., \& Kaufman, A. (1997). CollabCAD: A Toolkit for Integrated Synchronous and Asynchronous Sharing of CAD Applications. TeamCAD: GVU/NIST workshop on collaborative design, (pp. 131-137).

Panchal, J. H., Fernandez, M. G., Paredis, C. J., Allen, J. K., \& Mistree, F. (2007, September). An Interval-based Constraint Satisfaction (IBCS) Method for Decentralized, Collaborative Multifunctional Design. Concurrent Engineering: Research and Applications, 15, 309-323.

Park, T., \& Kim, K.-J. (1998). Determination of an Optimal Set of Design Requirements using House of Quality. Journal of Operations Management, 569-581.

POHL, J., CHAPMAN, C., \& POHL, K. J. (2000). Computer - Aided Design Systems for the 21st Century: Some Design. Nijkerk, The Netherlands: 5th International Conference on Design and Decision-Support Systems for Architecture and Urban.

Red, E., Holyoak, V., Jensen, C., Marshall, F., Ryskamp, J., \& Xu, Y. (2009). v-Cax: A Research Agenda for Collaboration Computer-aided Applications. Brigham Young University.

Ryskamp, J. D. (2010, December). Developing Intelligent Engineering Collaboration. BYU Department of Mechanical Engineering.

Ryskamp, J., Jenson, C. G., Mix, K., \& Red, E. (2010). Leveraging Design Rational to Improve Collaboration Co-design CAD Environments.

Sääksvuori, A., \& Immonen, A. (2008). Product Lifecycle Management. Berlin: Springer.

Sun, C., Xia, S., Sun, D., Chen, D., Shen, H., \& Cai, W. (2006, December). Transparent Adaptation of Single-User Applications for Multi-User Real-Time Collaboration. ACM Transactions on Computer-Human Interaction, 13(4), 531-582. 
Tang, D., Zheng, L., Li, Z., Li, D., \& Zhang, S. (2000). Re-engineering the Design Process for Concurrent Engineering. Computers and Industrial Engineering, 479-491.

Tay, F. E., \& Roy, A. (2003). CyberCAD: a collaborative approach in 3D-CAD technology in a multimedia-supported environment. Computers In Industry, 127-145.

Ulrich, K. T., \& Eppinger, S. (2004). Product Design and Development. New York: McGrawHill Companies, Inc.

Willingham, D. T. (2010, Summer). Have Technology and Multitasking Rewired How Students Learn? American Educator, pp. 23-28.

Woolley, A., Chabris, C., Pentland, A., Hashmi, N., \& Malone, T. (2010). Evidence for a Collective Intelligence Factor in the Performance of Human Groups. Science 330, 686688.

Zhang, H., Qiu, W., \& Zhang, H. (2006, December). An Approach to Measuring Coupled Tasks Strength and Sequencing of Coupled Tasks in New Product Development. CONCURRENT ENGINEERING: Research and Applications, 14(4).

Zheng, Y., Shen, H., \& Sun, C. (2008). Adapting Single-user AutoCAD System to Support Realtime Collaborative Design: Issues, Challenges and Achievements. 10th International Workshop on Collaborative Editing Systems, in conjunction with ACM CSCW 2008. San Diego. 